\title{
Bifurcation Analysis of Gene Regulatory Circuits Subject to Copy Number Variation*
}

\author{
Yuriy Mileyko ${ }^{\dagger}$ and Joshua S. Weitz
}

\begin{abstract}
Gene regulatory networks are comprised of many small gene circuits. Understanding expression dynamics of gene circuits for broad ranges of parameter space may provide insight into the behavior of larger regulatory networks as well as facilitate the use of circuits as autonomous units performing specific regulatory tasks. In this paper, we consider three common gene circuits and investigate the dependence of gene expression dynamics on the circuit copy number. In particular, we perform a detailed bifurcation analysis of the circuits' corresponding nonlinear gene regulatory models restricted to protein-only dynamics. Employing a geometric approach to bifurcation theory, we are able to derive closed form expressions for conditions which guarantee existence of saddle-node bifurcations caused by variation in the circuit copy number or copy number concentration. This result shows that the drastic effect of copy number variation on equilibrium behavior of gene circuits is highly robust to variation in other parameters in the circuits. We discuss a possibility of extending the current results to higher dimensional models which incorporate more details of the gene regulatory process.
\end{abstract}

Key words. copy number variation, bifurcation theory, regulatory networks

AMS subject classifications. $34 \mathrm{C} 23,34 \mathrm{C} 60,37 \mathrm{G} 10$

DOI. $10.1137 / 090771247$

1. Introduction. Understanding and predicting the quantitative expression dynamics of gene regulatory networks is a fundamental problem in biology [15]. In particular, it is often desirable to foresee how variation in parts of or inputs to a network leads to significant changes in network dynamics. However, the large number of genes, molecular species, interactions, and kinetic parameters in gene regulatory networks makes the general problem extremely difficult. Experiments that monitor gene regulation permit simultaneous measurement of the dynamics of many gene products. Newer technologies (like next-generation sequencing-based efforts to monitor transcription) can simultaneously track the dynamics of thousands of genes, producing unprecedented time series data regarding gene regulatory dynamics [3]. Given the complexity of the state space, directing experiments to probe important/useful regions of parameter space requires having some expectations regarding the outcome of dynamics. Hence, employing quantitative approaches to obtaining generalized insight into the intricacies of expression dynamics is essential (see, e.g., [10]).

There have been numerous attempts to model and simulate dynamics of gene regulatory

\footnotetext{
* Received by the editors September 15, 2009; accepted for publication (in revised form) by J. Collins April 16, 2010; published electronically July 6, 2010. This research was supported by the Defense Advanced Research Projects Agency under grant HR0011-05-1-0057 and the James S. McDonnell Foundation.

http://www.siam.org/journals/siads/9-3/77124.html

${ }^{\dagger}$ Department of Mathematics, Duke University, Durham, NC 27708 (yury@math.duke.edu).

${ }^{\ddagger}$ Schools of Biology and Physics, Georgia Institute of Technology, Atlanta, GA 30332 (jsweitz@gatech.edu). This author holds a Career Award at the Scientific Interface from the Burroughs Wellcome Fund.
} 
networks (see $[9,1,15]$ for reviews). The classes of models include Boolean-based approaches, algebraic models, ordinary differential equation (ODE) models, stochastic differential equation models, and stochastic kinetics simulators. Of these, the most common approach is to numerically simulate the model directly, often using Monte Carlo techniques [18, 21]. Such approaches can be computationally expensive and very often are not well suited to study dependence of the network dynamics on various parameters. For instance, it may be important to know not only that a qualitative change in dynamics is possible as we vary a specific parameter, but also the robustness of such a change with respect to the other parameters: if the change in dynamics happens only for a very small region of parameter values, then it might be biologically irrelevant. Promising methods exist, such as those based on the Conley index [4], to resolve the general problem of predicting qualitative dependence of nonlinear dynamic models on parameter values. However, such methods are also computationally intensive, and possible transitions in dynamics that can be obtained with such a method are not yet well documented. Besides, since the number of parameters involved in regulatory networks is very large even for small networks, computational approaches to the robustness problem might suffer from the curse of dimensionality.

While analytical approaches to analyzing parameter dependence of expression dynamics of large-scale regulatory networks are virtually nonexistent, very small networks might still be amenable to analysis using the classical approach based on continuous dynamical systems $[13,24]$. Fortunately, a large regulatory network is composed of many gene circuits, i.e., small, often overrepresented, subgraphs of the network [1]. Understanding the dynamics of such circuits may give us insight into the dynamics of larger networks.

In this paper we analyze expression dynamics of three common gene circuits: positive feedback, bistable feedback, and toggle switch circuits. In particular, we explain what qualitative changes may happen to the expression dynamics of gene circuits as we vary a specific network parameter - the circuit copy number - and how robust these changes are with respect to other parameters involved in the systems. The biological importance of copy number variation $(\mathrm{CNV})$ and its effect on phenotype is widely recognized. CNV is thought to be the dominant cause of phenotypic evolution [17] and has been implicated in the onset of diseases, including cancer [20]. Two recent papers introduced the notion that the dynamic impact of CNV can be understood, in part, by considering the nonlinear effect of gene expression as a function of changes in gene copy number (or gene copy number concentration) [25, 19]. Here, we perform a detailed bifurcation analysis of the dynamical systems representing the three circuits and derive closed form expressions for conditions which guarantee existence of bifurcations that may lead to drastic changes in expression dynamics. In doing so, we utilize both classical and geometric approaches to bifurcation theory $[22,23,16,5,6]$.

The paper is structured as follows. In section 2 we describe the circuits and the corresponding differential equations models. Investigation of equilibrium dynamics of the models is done in section 3. Section 4 contains the main results of the paper-derivation of conditions which guarantee a possibility of drastic changes in circuits' equilibrium dynamics caused by CNV. Note that derivation of these conditions relies heavily upon Lemma 3.8 (presented near the end of section 3), which recasts classical conditions for structural instability in geometric terms. Section 5 concludes the paper with a brief discussion of our results and their extensibility to more accurate, higher dimensional models of gene circuits.

Copyright (c) by SIAM. Unauthorized reproduction of this article is prohibited. 

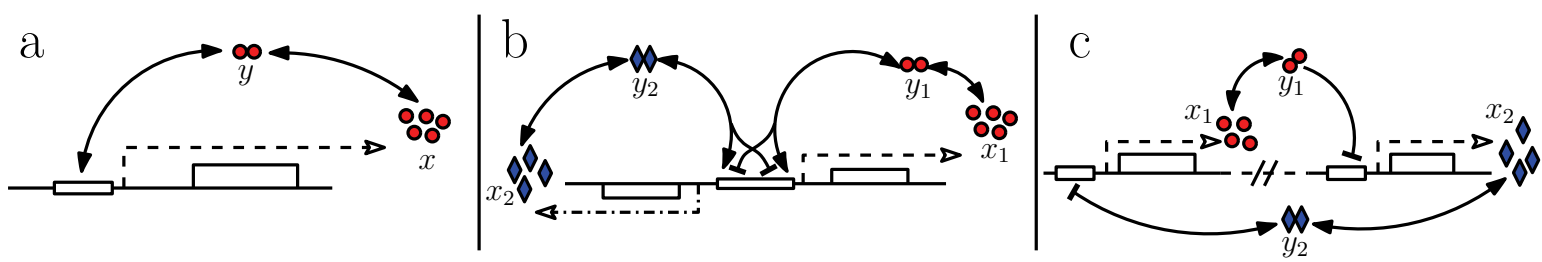

Figure 1. Schematic diagrams of gene circuits: (a) Positive feedback circuit; (b) bistable feedback circuit; (c) toggle switch circuit. In line with (2.1)-(2.3), monomers are marked with $x$ for the positive feedback circuit and with $x_{1}, x_{2}$ for the bistable feedback and toggle switch circuits. Dimers are marked with $y$ and $y_{1}, y_{2}$, respectively. Note that dashed lines denote transcription/translation, whereas solid lines denote protein (un)binding and (de)dimerization events (adapted from [19]).

2. Motifs and their differential equation models. We shall now introduce the three gene circuits and the corresponding differential equation models.

The first gene circuit is the positive feedback circuit (see [14, 12]), whose schematic diagram is shown in Figure 1(a). This circuit is comprised of a single gene whose sequence is first transcribed into mRNA and then translated into a protein. The protein can dimerize and bind to the promoter, resulting in an enhanced transcription rate. The dynamics of such a system can be described by the following equations:

$$
\begin{aligned}
\dot{x} & =2 \kappa_{-} y-2 \kappa_{+} x^{2}+\sigma m-\gamma_{x} x \\
\dot{y} & =-\kappa_{-} y+\kappa_{+} x^{2}+k_{-} d_{y}-k_{+} d_{0} y \\
\dot{d_{0}} & =k_{-} d_{y}-k_{+} d_{0} y \\
\dot{d_{y}} & =k_{+} d_{0} y-k_{-} d_{y} \\
\dot{m} & =\alpha d_{0}+\beta d_{y}-\gamma_{m} m
\end{aligned}
$$

where $x$ and $y$ denote the concentrations of the protein monomers and dimers, respectively, $d_{0}$ and $d_{y}$ are the concentrations of unoccupied and occupied promoters, and $m$ is the mRNA concentration. Parameters $\alpha$ and $\beta$ are the basal and enhanced transcription rates, $\kappa_{ \pm}$denote the dimerization and undimerization rates, $k_{ \pm}$are the binding and dissociation rates of the dimers to the promoter site, $\gamma_{x}$ and $\gamma_{m}$ are the degradation rates of the protein monomers and mRNA, respectively, and $\sigma$ is the translation rate. Notice also that the total number of promoters, $d=d_{y}+d_{0}$, is constant, and we define the dimensionless copy number as $\mathcal{N}=d / C$, where $C$ is the conversion factor denoting the concentration of a single molecule in a cell. Note that for simplicity and ease of presentation, we will use the term "copy number" to denote the parameter of interest. However, all results in this paper are formally derived in terms of copy number concentration, so both changes in gene copy number or in cell volume can have important physiological effects.

The bistable feedback circuit (Figure 1(b)) has two genes that share a promoter (see [8]). As in the previous case, the proteins produced by the two genes can dimerize and bind to the promoter. In addition to enhancing (or inhibiting) the transcription rate of its own gene, the bound protein completely shuts down transcription of the other gene. Equations describing

Copyright (c) by SIAM. Unauthorized reproduction of this article is prohibited. 
the dynamics can be written as

$$
\begin{aligned}
\dot{x_{i}} & =2 \kappa_{-} y_{i}-2 \kappa_{+} x_{i}^{2}+\sigma m_{i}-\gamma_{i} x_{i}, \\
\dot{y_{i}} & =-\kappa_{-} y_{i}+\kappa_{+} x_{i}^{2}+k_{-} d_{i}-k_{+} d_{0} y_{i}, \\
\dot{d}_{0} & =k_{-}\left(d_{1}+d_{2}\right)-k_{+} d_{0}\left(y_{1}+y_{2}\right), \\
\dot{d}_{i} & =k_{+} d_{0} y_{i}-k_{-} d_{i}, \\
\dot{m}_{i} & =\alpha_{i} d_{0}+\beta_{i} d_{i}-\gamma_{m} m_{i},
\end{aligned}
$$

where $i=1,2, x_{i}$ and $y_{i}$ are the concentrations of monomers and dimers, respectively, $d_{0}$ is the concentration of unoccupied promoters, $d_{i}$ are the concentrations of promoters occupied by the first and the second proteins, and $m_{i}$ are the mRNA concentrations. Parameters $\alpha_{i}$ and $\beta_{i}$ are the basal and enhanced transcription rates; $\gamma_{i}$ are the degradation rates. The rest of the parameters are the same as in the model for the positive feedback circuit. The total concentration of promoters is $d=d_{0}+d_{1}+d_{2}$.

Last, we consider the toggle switch circuit (see Figure 1(c)), which has two genes with separate promoters. In this case dimers of a protein bind to the promoter of the other gene and inhibit (although not completely) its transcription rate (compare to [11]). Equations describing its dynamics are

$$
\begin{aligned}
\dot{x_{i}} & =2 \kappa_{-} y_{i}-2 \kappa_{+} x_{i}^{2}+\sigma m_{i}-\gamma_{i} x_{i}, \\
\dot{y_{i}} & =-\kappa_{-} y_{i}+\kappa_{+} x_{i}^{2}+k_{-} d_{i+1}-k_{+} d_{0, i+1} y_{i}, \\
\dot{d}_{0, i} & =k_{-} d_{i}-k_{+} d_{0, i} y_{i+1} \\
\dot{d_{i}} & =k_{+} d_{0, i} y_{i+1}-k_{-} d_{i}, \\
\dot{m_{i}} & =\alpha_{i} d_{0, i}+\beta_{i} d_{i}-\gamma_{m} m_{i},
\end{aligned}
$$

where $i=1,2$, and to simplify notation we assume that $i \in 1+\mathbb{Z}_{2}$; that is, the index wraps back to 1 once it becomes greater than 2 . The variables and parameters have the same meaning as in the case of the bistable circuit, except that $d_{0, i}$ and $d_{i}$ denote free and occupied promoters of the $i$ th gene and $\beta_{i}$ are inhibited rather than enhanced transcription rates. The total concentration of promoters is $d=d_{0,1}+d_{1}=d_{0,2}+d_{2}$.

As demonstrated in [19], equilibrium dynamics of these models can depend sensitively on the gene copy number. The main goal of this paper is to find conditions under which CNV will lead to drastic changes in the circuits' equilibrium dynamics. Since only nonnegative values of the variables are biologically relevant, we shall consider dynamics of the above models only in the first orthant, which is, coincidentally, an invariant set for each of the models.

A complete analysis of equilibrium dynamics of higher dimensional systems like (2.1)-(2.3) is often not feasible. However, some reactions involved in the systems are much faster than others creating a time scale separation. Therefore, it is quite common to simplify these systems by constructing their quasi-steady-state approximations, that is, assuming that all but the "slowest" variables are in their steady states [7]. It is known, for example, that translation of proteins is slower than transcription and much slower than (de)dimerization and (un)binding. Thus, systems (2.1), (2.2), and (2.3) can be simplified to (see [19])

$$
\dot{u}=\mathcal{N} \frac{\hat{\alpha}+\hat{\beta} u^{2}}{1+u^{2}}-\gamma_{p} u,
$$

Copyright (c) by SIAM. Unauthorized reproduction of this article is prohibited. 


$$
\dot{u}_{i}=\mathcal{N} \frac{\hat{\alpha}_{i}+\hat{\beta}_{i} u_{i}^{2}}{1+u_{1}^{2}+u_{2}^{2}}-\gamma_{i} u_{i}, \quad i=1,2
$$

and

$$
\dot{u}_{i}=\mathcal{N} \frac{\hat{\alpha}_{i}+\hat{\beta}_{i} u_{i+1}^{2}}{1+u_{i+1}^{2}}-\gamma_{i} u_{i}, \quad i \in 1+\mathbb{Z}_{2},
$$

respectively. We have rescaled the variables and parameters as follows. Let $c_{p}=\frac{\kappa_{+}}{\kappa_{-}}$and $c_{d}=\frac{k_{+}}{k_{-}}$. Then

and

$$
u=\sqrt{c_{p} c_{d}} x, \quad \hat{\alpha}=\frac{C \alpha \sigma \sqrt{c_{p} c_{d}}}{\gamma_{m}}, \quad \hat{\beta}=\frac{C \beta \sigma \sqrt{c_{p} c_{d}}}{\gamma_{m}}
$$

$$
u_{i}=\sqrt{c_{p} c_{d}} x_{i}, \quad \hat{\alpha}_{i}=\frac{C \alpha_{i} \sigma \sqrt{c_{p} c_{d}}}{\gamma_{m}}, \quad \hat{\beta}_{i}=\frac{C \beta_{i} \sigma \sqrt{c_{p} c_{d}}}{\gamma_{m}} .
$$

Quasi-steady-state approximations may, in general, lead to incorrect equilibrium dynamics. However, the protein-only models that we derived above have become de facto standard models for the circuits' expression dynamics. Hence, we shall use systems (2.4)-(2.6) as our initial models. We make the claim in section 5 that most of our analysis can be extended to the case of higher dimensional systems (2.1)-(2.3).

3. Equilibrium dynamics. We now proceed to investigate possible equilibrium dynamics of systems (2.4)-(2.6) and dependence of these dynamics on CNV. First, let us define functions $F_{p f}: \mathbb{R}^{2} \rightarrow \mathbb{R}, F_{b f}: \mathbb{R}^{3} \rightarrow \mathbb{R}^{2}$, and $F_{t s}: \mathbb{R}^{3} \rightarrow \mathbb{R}^{2}$ as follows:

$$
F_{p f}(\bar{u}, \mathcal{N})=f_{p f}(\bar{u})-\mathcal{N}, \quad f_{p f}=\frac{\bar{u}\left(1+\bar{u}^{2}\right)}{\bar{\alpha}+\bar{\beta} \bar{u}^{2}}
$$

where $\bar{\alpha}=\frac{\hat{\alpha}}{\gamma_{p}}, \bar{\beta}=\frac{\hat{\beta}}{\gamma_{p}} ;$

$$
\begin{aligned}
F_{b f}\left(\bar{u}_{1}, \bar{u}_{2}, \mathcal{N}\right) & =\left(f_{b f}\left(\bar{u}_{1}, \bar{u}_{2}\right)-\mathcal{N}, g_{b f}\left(\bar{u}_{1}, \bar{u}_{2}\right)\right), \\
f_{b f}\left(\bar{u}_{1}, \bar{u}_{2}\right) & =\frac{\bar{u}_{1}\left(1+\bar{u}_{1}^{2}\right)}{\bar{\alpha}_{1}+\bar{\beta}_{1} \bar{u}_{1}^{2}}+\frac{\bar{u}_{2}^{3}}{\bar{\alpha}_{2}+\bar{\beta}_{2} \bar{u}_{2}^{2}}, \\
g_{b f}\left(\bar{u}_{1}, \bar{u}_{2}\right) & =\frac{\bar{u}_{1}}{\bar{\alpha}_{1}+\bar{\beta}_{1} \bar{u}_{1}^{2}}-\frac{\bar{u}_{2}}{\bar{\alpha}_{2}+\bar{\beta}_{2} \bar{u}_{2}^{2}},
\end{aligned}
$$

where $\bar{\alpha}_{i}=\frac{\hat{\alpha}_{i}}{\gamma_{i}}, \bar{\beta}_{i}=\frac{\hat{\beta}_{i}}{\gamma_{i}}, i=1,2$; and

$$
\begin{aligned}
& F_{t s}\left(\bar{u}_{1}, \bar{u}_{2}, \mathcal{N}\right)=\left(f_{t s}\left(\bar{u}_{1}, \bar{u}_{2}\right)-\mathcal{N}, g_{t s}\left(\bar{u}_{1}, \bar{u}_{2}\right)\right), \\
& f_{t s}\left(\bar{u}_{1}, \bar{u}_{2}\right)=\frac{\bar{u}_{1}\left(1+\bar{u}_{2}^{2}\right)}{\bar{\alpha}_{1}+\bar{\beta}_{1} \bar{u}_{2}^{2}}, \\
& g_{t s}\left(\bar{u}_{1}, \bar{u}_{2}\right)=\frac{\bar{u}_{1}\left(\bar{\alpha}_{2}+\bar{\beta}_{2} \bar{u}_{1}^{2}\right)}{1+\bar{u}_{1}^{2}}-\frac{\bar{u}_{2}\left(\bar{\alpha}_{1}+\bar{\beta}_{1} \bar{u}_{2}^{2}\right)}{1+\bar{u}_{2}^{2}} .
\end{aligned}
$$

Copyright (c) by SIAM. Unauthorized reproduction of this article is prohibited. 
An easy computation shows that the steady states of systems (2.4), (2.5), and (2.6) are solutions to the equations $F_{p f}=0, F_{b f}=0$, and $F_{t s}=0$, respectively.

Now we can prove the following theorem.

Theorem 3.1. There exists $\varepsilon>0$ such that for every $0<\mathcal{N}<\varepsilon$ each of the systems (2.4)-(2.6) has a unique, stable steady state.

Proof. First, let us consider (2.4). Notice that $\mathcal{N} \rightarrow 0$ implies that each steady state $\bar{u} \rightarrow 0$. It is also evident that the derivative of $f_{p f}$,

$$
\frac{\mathrm{d} f_{p f}}{\mathrm{~d} \bar{u}}=\frac{\bar{\beta} \bar{u}^{4}-(\bar{\beta}-3 \bar{\alpha}) \bar{u}^{2}+\bar{\alpha}}{\left(\bar{\alpha}+\bar{\beta} \bar{u}^{2}\right)^{2}}
$$

is positive for small enough $\bar{u}$. Hence, $\exists \delta>0$ such that $f_{p f}$ is monotonic for $\bar{u} \in(0, \delta)$, and we can pick $\varepsilon$ such that the equation $\mathcal{N}=f_{p f}(\bar{u})$ has a unique solution if $\mathcal{N}<\varepsilon$. The derivative of the right-hand side of (2.4) at the steady state is given by

$$
J_{p f}=\mathcal{N} \frac{2(\hat{\beta}-\hat{\alpha}) \bar{u}}{\left(1+\bar{u}^{2}\right)^{2}}-\gamma_{p}
$$

It is clearly negative if $\mathcal{N}$ and $\bar{u}$ are small enough. Thus, stability of the steady state can be guaranteed by adjusting the choice of $\varepsilon$.

System (2.6) can be analyzed in a very similar way. Again, $\mathcal{N} \rightarrow 0$ implies that each steady state $\left(\bar{u}_{1}, \bar{u}_{2}\right) \rightarrow(0,0)$. Notice that $\exists \delta_{1}>0$ such that $\frac{\partial g_{s}}{\partial \bar{u}_{1}} \neq 0$ for $\bar{u}_{1} \in\left(0, \delta_{1}\right)$. Therefore, there exists a smooth, positive function $h_{t s}\left(\bar{u}_{2}\right)$ such that $g_{t s}\left(h_{t s}\left(\bar{u}_{2}\right), \bar{u}_{2}\right) \equiv 0$ in some neighborhood of the origin. Moreover, $h_{t s}\left(\bar{u}_{2}\right) \rightarrow 0$ as $\bar{u}_{2} \rightarrow 0$ and

$$
\frac{\mathrm{d} h_{t s}}{\mathrm{~d} \bar{u}_{2}}=\frac{\left(\bar{u}_{2}^{4}+\left(3-a_{1}\right) \bar{u}_{2}^{2}+a_{1}\right)\left(1+\bar{u}_{1}^{2}\right)^{2}}{\left(\bar{u}_{1}^{4}+\left(3-a_{2}\right) \bar{u}_{1}^{2}+a_{2}\right)\left(1+\bar{u}_{2}^{2}\right)^{2}}>c>0
$$

for small enough $\bar{u}_{1}$ and $\bar{u}_{2}$, where $c$ is some small positive constant.

Now notice that steady states of system (2.6) are defined by solutions to the equation $\mathcal{N}=f_{t s}\left(h_{t s}\left(\bar{u}_{2}\right), \bar{u}_{2}\right)$. In addition,

$$
\frac{\mathrm{d} f_{t s}}{\mathrm{~d} \bar{u}_{2}}=\frac{\mathrm{d} h_{t s}}{\mathrm{~d} \bar{u}_{2}} \frac{\left(1+\bar{u}_{2}^{2}\right)}{\bar{\alpha}_{1}+\bar{\beta}_{1} \bar{u}_{2}^{2}}+2 h_{t s}\left(\bar{u}_{2}\right) \frac{\left(\bar{\alpha}_{1}-\bar{\beta}_{1}\right) \bar{u}_{2}}{\left(\bar{\alpha}_{1}+\bar{\beta}_{1} \bar{u}_{2}^{2}\right)^{2}}>0
$$

for small enough $\bar{u}_{2}$. Thus, $f_{t s}\left(h_{t s}\left(\bar{u}_{2}\right), \bar{u}_{2}\right)$ is a monotonic function of $\bar{u}_{2}$, and there is a unique solution to the above equation if $\mathcal{N}<\varepsilon$ for some small $\varepsilon$. The Jacobian of system (2.6) at this steady state is given by

$$
J_{t s}=\left(\begin{array}{cc}
-\gamma_{1} & 2 \mathcal{N} \frac{\left(\hat{\beta}_{1}-\hat{\alpha}_{1}\right) \bar{u}_{2}}{\left(1+\bar{u}_{2}^{2}\right)^{2}} \\
2 \mathcal{N} \frac{\left(\hat{\beta}_{2}-\hat{\alpha}_{2}\right) \bar{u}_{1}}{\left(1+\bar{u}_{1}^{2}\right)^{2}} & -\gamma_{2}
\end{array}\right) .
$$

Its trace $\operatorname{tr}\left(J_{t s}\right)=-\left(\gamma_{1}+\gamma_{2}\right)<0$. Its determinant

$$
\operatorname{det}\left(J_{t s}\right)=\gamma_{1} \gamma_{2}-4 \mathcal{N}^{2} \frac{\left(\hat{\beta}_{1}-\hat{\alpha}_{1}\right)\left(\hat{\beta}_{2}-\hat{\alpha}_{2}\right) \bar{u}_{1} \bar{u}_{2}}{\left(1+\bar{u}_{1}^{2}\right)^{2}\left(1+\bar{u}_{2}^{2}\right)^{2}}
$$

Copyright (c) by SIAM. Unauthorized reproduction of this article is prohibited. 
Picking $\varepsilon$ small enough and having $\mathcal{N}<\varepsilon$ leads to such small $\bar{u}_{1}$ and $\bar{u}_{2}$ that $\operatorname{det}\left(J_{t s}\right)>0$. Hence the real parts of the eigenvalues

$$
\lambda_{ \pm}=\operatorname{tr}\left(J_{t s}\right) \pm \sqrt{\operatorname{tr}\left(J_{t s}\right)^{2}-4 \operatorname{det}\left(J_{t s}\right)}
$$

are negative and the steady state is stable.

The proof for the case of system (2.5) follows the same steps as in the previous case. Once again, we can see that $\mathcal{N} \rightarrow 0$ implies that each steady state $\left(\bar{u}_{1}, \bar{u}_{2}\right) \rightarrow(0,0)$. There is also a smooth, positive function $h_{b f}\left(\bar{u}_{1}\right)$ such that $g_{b f}\left(\bar{u}_{1}, h_{b f}\left(\bar{u}_{1}\right)\right) \equiv 0$ in a neighborhood of the origin, $h_{b f}\left(\bar{u}_{1}\right) \rightarrow 0$ as $\bar{u}_{1} \rightarrow 0$, and

$$
0>\frac{\mathrm{d} h_{b f}}{\mathrm{~d} \bar{u}_{1}}=-\frac{\left(\bar{\alpha}_{1}-\bar{\beta}_{1} \bar{u}_{1}^{2}\right)\left(\bar{\alpha}_{2}+\bar{\beta}_{2} \bar{u}_{2}^{2}\right)^{2}}{\left(\bar{\alpha}_{2}-\bar{\beta}_{2} \bar{u}_{2}^{2}\right)\left(\bar{\alpha}_{1}+\bar{\beta}_{1} \bar{u}_{1}^{2}\right)^{2}}>-c
$$

for small $\bar{u}_{1}$ and $\bar{u}_{2}$, where $c$ is some positive constant. The steady states are now defined by solutions to the equation $\mathcal{N}=f_{b f}\left(\bar{u}_{1}, h_{b f}\left(\bar{u}_{1}\right)\right)$, and

$$
\frac{\mathrm{d} f_{b f}}{\mathrm{~d} \bar{u}_{1}}=\frac{\bar{\beta}_{1} \bar{u}_{1}^{4}-\left(\bar{\beta}_{1}-3 \bar{\alpha}_{1}\right) \bar{u}_{1}^{2}+\bar{\alpha}_{1}}{\left(\bar{\alpha}_{1}+\bar{\beta}_{1} \bar{u}_{1}^{2}\right)^{2}}+\frac{\mathrm{d} h_{b f}}{\mathrm{~d} \bar{u}_{1}} \frac{h_{b f}\left(\bar{u}_{1}\right)^{2}\left(\bar{\beta}_{2} h_{b f}\left(\bar{u}_{1}\right)^{2}+\bar{\alpha}_{2}\right)}{\left(\bar{\alpha}_{2}+\bar{\beta}_{2} h_{b f}\left(\bar{u}_{1}\right)^{2}\right)^{2}}>0
$$

for small $\bar{u}_{1}$. Thus, $f_{b f}\left(\bar{u}_{1}, h_{b f}\left(\bar{u}_{1}\right)\right)$ is a monotonic function of $\bar{u}_{1}$, and there is a unique solution to the above equation if $\mathcal{N}<\varepsilon$ for some small $\varepsilon$. The Jacobian of (2.5) at this steady state is given by

$$
J_{b f}=\left(\begin{array}{cc}
2 \mathcal{N} \frac{\left(\hat{\beta}_{1}-\hat{\alpha}_{1}+\hat{\beta}_{1} \bar{u}_{2}^{2}\right) \bar{u}_{1}}{\left(1+\bar{u}_{1}^{2}+\bar{u}_{2}^{2}\right)^{2}}-\gamma_{1} & -2 \mathcal{N} \frac{\bar{u}_{2}\left(\hat{\alpha}_{1}+\hat{\beta}_{1} \bar{u}_{1}^{2}\right)}{\left(1+\bar{u}_{1}^{2}+\bar{u}_{2}^{2}\right)^{2}} \\
-2 \mathcal{N} \frac{\bar{u}_{1}\left(\hat{\alpha}_{2}+\hat{\beta}_{2} \bar{u}_{2}^{2}\right)}{\left(1+\bar{u}_{1}^{2}+\bar{u}_{2}^{2}\right)^{2}} & 2 \mathcal{N} \frac{\left(\hat{\beta}_{2}-\hat{\alpha}_{2}+\hat{\beta}_{2} \bar{u}_{1}^{2}\right) \bar{u}_{2}}{\left(1+\bar{u}_{1}^{2}+\bar{u}_{2}^{2}\right)^{2}}-\gamma_{2}
\end{array}\right) .
$$

The trace

$$
\operatorname{tr}\left(J_{b f}\right)=-\left(\gamma_{1}+\gamma_{2}\right)+2 \mathcal{N} \frac{\left(\hat{\beta}_{1}-\hat{\alpha}_{1}+\hat{\beta}_{1} \bar{u}_{2}^{2}\right) \bar{u}_{1}+\left(\hat{\beta}_{2}-\hat{\alpha}_{2}+\hat{\beta}_{2} \bar{u}_{1}^{2}\right) \bar{u}_{2}}{\left(1+\bar{u}_{1}^{2}+\bar{u}_{2}^{2}\right)^{2}}
$$

and the determinant

$$
\begin{aligned}
\operatorname{det}\left(J_{b f}\right) & =\gamma_{1} \gamma_{2}-2 \mathcal{N} \frac{\gamma_{2} \bar{u}_{1}\left(\hat{\beta}_{1}-\hat{\alpha}_{1}+\hat{\beta}_{1} \bar{u}_{2}^{2}\right)+\gamma_{1} \bar{u}_{2}\left(\hat{\beta}_{2}-\hat{\alpha}_{2}+\hat{\beta}_{2} \bar{u}_{1}^{2}\right)}{\left(1+\bar{u}_{1}^{2}+\bar{u}_{2}^{2}\right)^{2}} \\
& +4 \mathcal{N}^{2} \frac{\bar{u}_{2} \bar{u}_{1}\left[\left(\hat{\beta}_{1}-\hat{\alpha}_{1}+\hat{\beta}_{1} \bar{u}_{2}^{2}\right)\left(\hat{\beta}_{2}-\hat{\alpha}_{2}+\hat{\beta}_{2} \bar{u}_{1}^{2}\right)-\left(\hat{\alpha}_{1}+\hat{\beta}_{1} \bar{u}_{1}^{2}\right)\left(\hat{\alpha}_{2}+\hat{\beta}_{2} \bar{u}_{2}^{2}\right)\right]}{\left(1+\bar{u}_{1}^{2}+\bar{u}_{2}^{2}\right)^{4}} .
\end{aligned}
$$

We can see now that it is possible to pick $\varepsilon$ such that $\mathcal{N}<\varepsilon$ makes $\bar{u}_{1}$ and $\bar{u}_{2}$ so small that $\operatorname{tr}\left(J_{b f}\right)<0$ and $\operatorname{det}\left(J_{b f}\right)>0$. Consequently, the real parts of the eigenvalues are negative, and the steady state is stable.

Interestingly, large values of the copy number give a similar picture, at least for the positive feedback and toggle switch circuits.

Copyright (C) by SIAM. Unauthorized reproduction of this article is prohibited. 
Theorem 3.2. There exists $E>0$ such that for every $\mathcal{N}>E$ systems (2.4) and (2.6) have a unique, stable steady state. System (2.5) has three steady states in this case, two of which are stable.

Proof. The proof of uniqueness in the case of systems (2.4) and (2.6) is largely the same as in Theorem 3.1, and we leave it to the reader. We merely notice that $\mathcal{N} \rightarrow \infty$ implies $\bar{u} \rightarrow \infty$ and $\left(\bar{u}_{1}, \bar{u}_{2}\right) \rightarrow(\infty, \infty)$ for positive feedback and toggle switch, respectively. To prove stability in the case of system (2.4) we rewrite (3.9) as

$$
J_{p f}=2 \frac{(\hat{\beta}-\hat{\alpha}) \bar{u}^{2}}{\left(1+\bar{u}^{2}\right)\left(\bar{\alpha}+\bar{\beta} \bar{u}^{2}\right)}-\gamma_{p} .
$$

Clearly, $J_{p f}<0$ if $\bar{u}$ is large enough, so the steady state is stable for large $\mathcal{N}$.

Stability of the steady state of system (2.6) is proved similarly. Rewrite the determinant of the Jacobian as

$$
\operatorname{det}\left(J_{t s}\right)=\gamma_{1} \gamma_{2}-4 \frac{\left(\hat{\beta}_{1}-\hat{\alpha}_{1}\right)\left(\hat{\beta}_{2}-\hat{\alpha}_{2}\right) \bar{u}_{1}^{2} \bar{u}_{2}^{2}}{\left(1+\bar{u}_{1}^{2}\right)\left(1+\bar{u}_{2}^{2}\right)\left(\bar{\alpha}_{1}+\bar{\beta}_{1} \bar{u}_{2}^{2}\right)\left(\bar{\alpha}_{2}+\bar{\beta}_{2} \bar{u}_{1}^{2}\right)} .
$$

Notice that $\operatorname{det}\left(J_{t s}\right)>0$ if $\bar{u}_{1}$ and $\bar{u}_{2}$ are large enough, so the steady state is stable for large $\mathcal{N}$.

The case of system (2.5) requires a little more algebra. First, notice that the equation $g_{b f}=0$ holds iff

or

$$
\bar{u}_{2}=h_{+}\left(\bar{u}_{1}\right)=\frac{\bar{\alpha}_{1}+\bar{\beta}_{1} \bar{u}_{1}^{2}+\sqrt{\left(\bar{\alpha}_{1}+\bar{\beta}_{1} \bar{u}_{1}^{2}\right)^{2}-4 \bar{\alpha}_{2} \bar{\beta}_{2} \bar{u}_{1}^{2}}}{2 \bar{\beta}_{2} \bar{u}_{1}}
$$

$$
\bar{u}_{2}=h_{-}\left(\bar{u}_{1}\right)=\frac{2 \bar{\alpha}_{2} \bar{u}_{1}}{\bar{\alpha}_{1}+\bar{\beta}_{1} \bar{u}_{1}^{2}+\sqrt{\left(\bar{\alpha}_{1}+\bar{\beta}_{1} \bar{u}_{1}^{2}\right)^{2}-4 \bar{\alpha}_{2} \bar{\beta}_{2} \bar{u}_{1}^{2}}} .
$$

Also notice that arbitrarily large values of $\mathcal{N}$ require that at least one of $\bar{u}_{i}, i=1,2$, be arbitrarily large.

Let $\bar{u}_{2}=h_{-}\left(\bar{u}_{1}\right)$. Clearly, $\bar{u}_{2}$ cannot be arbitrarily large in this case, so we have $\bar{u}_{1} \rightarrow \infty$ as $\mathcal{N} \rightarrow \infty$. Notice that $h_{-}\left(\bar{u}_{1}\right)$ is monotonically decreasing to 0 as $\bar{u}_{1} \rightarrow \infty$. Hence, $0>\frac{\mathrm{d} h_{-}}{\mathrm{d} \bar{u}_{1}}>-c$ for large $\bar{u}_{1}$, where $c$ is some positive constant (one can also show this by computing the derivative). Steady states in this case are defined by solutions to the equation $\mathcal{N}=f_{b f}\left(\bar{u}_{1}, h_{-}\left(\bar{u}_{1}\right)\right)$. Since

$$
\frac{\mathrm{d} f_{b f}}{\mathrm{~d} \bar{u}_{1}}=\frac{\bar{\beta}_{1} \bar{u}_{1}^{4}-\left(\bar{\beta}_{1}-3 \bar{\alpha}_{1}\right) \bar{u}_{1}^{2}+\bar{\alpha}_{1}}{\left(\bar{\alpha}_{1}+\bar{\beta}_{1} \bar{u}_{1}^{2}\right)^{2}}+\frac{\mathrm{d} h_{-}}{\mathrm{d} \bar{u}_{1}} \frac{h_{-}\left(\bar{u}_{1}\right)^{2}\left(\bar{\beta}_{2} h_{-}\left(\bar{u}_{1}\right)^{2}+\bar{\alpha}_{2}\right)}{\left(\bar{\alpha}_{2}+\bar{\beta}_{2} h_{-}\left(\bar{u}_{1}\right)^{2}\right)^{2}}>0
$$

for large $\bar{u}_{1}, f_{b f}\left(\bar{u}_{1}, h_{-}\left(\bar{u}_{1}\right)\right)$ is a monotonic function of $\bar{u}_{1}$. Hence there is a unique solution to the above equation if $\mathcal{N}>E$ for some large $E$.

Now let $\bar{u}_{2}=h_{+}\left(\bar{u}_{1}\right)$. Steady states in this case are defined by solutions to the equation $\mathcal{N}=f_{b f}\left(\bar{u}_{1}, h_{+}\left(\bar{u}_{1}\right)\right)$. Also,

$$
\frac{\mathrm{d} f_{b f}}{\mathrm{~d} \bar{u}_{1}}=\frac{\bar{\beta}_{1} \bar{u}_{1}^{4}-\left(\bar{\beta}_{1}-3 \bar{\alpha}_{1}\right) \bar{u}_{1}^{2}+\bar{\alpha}_{1}}{\left(\bar{\alpha}_{1}+\bar{\beta}_{1} \bar{u}_{1}^{2}\right)^{2}}+\frac{\mathrm{d} h_{+}}{\mathrm{d} \bar{u}_{1}} \frac{h_{+}\left(\bar{u}_{1}\right)^{2}\left(\bar{\beta}_{2} h_{+}\left(\bar{u}_{1}\right)^{2}+\bar{\alpha}_{2}\right)}{\left(\bar{\alpha}_{2}+\bar{\beta}_{2} h_{+}\left(\bar{u}_{1}\right)^{2}\right)^{2}},
$$

Copyright $\odot$ by SIAM. Unauthorized reproduction of this article is prohibited. 
and

$$
\frac{\mathrm{d} h_{+}}{\mathrm{d} \bar{u}_{1}}=-\frac{\bar{\alpha}_{1}}{2 \bar{\beta}_{2} \bar{u}_{1}^{2}}+\frac{\bar{\beta}_{1}}{2 \bar{\beta}_{2}}+\frac{\left(\frac{\bar{\alpha}_{1}}{2 \bar{\beta}_{2} \bar{u}_{1}}+\frac{\bar{\beta}_{1} \bar{u}_{1}}{2 \bar{\beta}_{2}}\right)\left(-\frac{\bar{\alpha}_{1}}{2 \bar{\beta}_{2} \bar{u}_{1}^{2}}+\frac{\bar{\beta}_{1}}{2 \bar{\beta}_{2}}\right)}{\sqrt{\left(\frac{\bar{\alpha}_{1}}{2 \bar{\beta}_{2} \bar{u}_{1}}+\frac{\bar{\beta}_{1} \bar{u}_{1}}{2 \bar{\beta}_{2}}\right)^{2}-\frac{\bar{\alpha}_{2}}{\bar{\beta}_{2}}}} .
$$

There are two cases to consider. First, suppose that $\bar{u}_{1} \rightarrow \infty$ as $\mathcal{N} \rightarrow \infty$. Then $h_{+}\left(\bar{u}_{1}\right) \rightarrow \infty$ as $\bar{u}_{1} \rightarrow \infty$. Also, $\frac{\mathrm{d} h_{+}}{\mathrm{d} \bar{u}_{1}}>0$ for large $\bar{u}_{1}$, which implies that $\frac{\mathrm{d} f_{b f}}{\mathrm{~d} \bar{u}_{1}}>0$ for large $\bar{u}_{1}$. Hence $f_{b f}\left(\bar{u}_{1}, h_{+}\left(\bar{u}_{1}\right)\right)$ is a monotonic function of $\bar{u}_{1}$, and there is a unique solution to the above equation if $\mathcal{N}>E$ for some large $E$ and $\bar{u}_{1}$ is large.

Now suppose that $\bar{u}_{1}$ is bounded for some arbitrarily large $\mathcal{N}$. Then we must have $\bar{u}_{2} \rightarrow \infty$ as $\mathcal{N} \rightarrow \infty$. Thus, we need $h_{+}\left(\bar{u}_{1}\right)$ to get arbitrarily large as $\mathcal{N} \rightarrow \infty$, which implies that $\bar{u}_{1}$ has to become arbitrarily small, that is, $\bar{u}_{1} \rightarrow 0$ as $\mathcal{N} \rightarrow \infty$. We can also see that $\frac{\mathrm{d} h_{+}}{\mathrm{d} \bar{u}_{1}} \rightarrow-\infty$ as $\bar{u}_{1} \rightarrow 0$, which implies that $\frac{\mathrm{d} f_{b f}}{\mathrm{~d} \bar{u}_{1}}<0$ for small $\bar{u}_{1}$. Hence, $f_{b f}\left(\bar{u}_{1}, h_{+}\left(\bar{u}_{1}\right)\right)$ is a monotonic function, and the equation $\mathcal{N}=f_{b f}\left(\bar{u}_{1}, h_{+}\left(\bar{u}_{1}\right)\right)$ has a unique solution if $\mathcal{N}>E$ for some large $E$ and $\bar{u}_{1}$ is small.

We now show that the steady state for which $\left(\bar{u}_{1}, \bar{u}_{2}\right) \rightarrow(0, \infty)$ as $\mathcal{N} \rightarrow \infty$ as well as the steady state for which $\left(\bar{u}_{1}, \bar{u}_{2}\right) \rightarrow(\infty, 0)$ as $\mathcal{N} \rightarrow \infty$ are stable for large enough $\mathcal{N}$. Rewrite the trace and the determinant of the Jacobian as follows:

$$
\begin{aligned}
\operatorname{tr}\left(J_{b f}\right)= & -\left(\gamma_{1}+\gamma_{2}\right)+2\left(\frac{\left(\hat{\beta}_{1}-\hat{\alpha}_{1}+\hat{\beta}_{1} \bar{u}_{2}^{2}\right) \bar{u}_{1}^{2}}{\left(1+\bar{u}_{1}^{2}+\bar{u}_{2}^{2}\right)\left(\bar{\alpha}_{1}+\bar{\beta}_{1} \bar{u}_{1}^{2}\right)}+\frac{\left(\hat{\beta}_{2}-\hat{\alpha}_{2}+\hat{\beta}_{2} \bar{u}_{1}^{2}\right) \bar{u}_{2}^{2}}{\left(1+\bar{u}_{1}^{2}+\bar{u}_{2}^{2}\right)\left(\bar{\alpha}_{2}+\bar{\beta}_{2} \bar{u}_{2}^{2}\right)}\right) \\
\operatorname{det}\left(J_{b f}\right) & =\gamma_{1} \gamma_{2}-2\left(\frac{\gamma_{2} \bar{u}_{1}^{2}\left(\hat{\beta}_{1}-\hat{\alpha}_{1}+\hat{\beta}_{1} \bar{u}_{2}^{2}\right)}{\left(1+\bar{u}_{1}^{2}+\bar{u}_{2}^{2}\right)\left(\bar{\alpha}_{1}+\bar{\beta}_{1} \bar{u}_{1}^{2}\right)}+\frac{\gamma_{1} \bar{u}_{2}^{2}\left(\hat{\beta}_{2}-\hat{\alpha}_{2}+\hat{\beta}_{2} \bar{u}_{1}^{2}\right)}{\left(1+\bar{u}_{1}^{2}+\bar{u}_{2}^{2}\right)\left(\bar{\alpha}_{2}+\bar{\beta}_{2} \bar{u}_{2}^{2}\right)}\right) \\
& +4 \frac{\bar{u}_{2}^{2} \bar{u}_{1}^{2}\left[\left(\hat{\beta}_{1}-\hat{\alpha}_{1}+\hat{\beta}_{1} \bar{u}_{2}^{2}\right)\left(\hat{\beta}_{2}-\hat{\alpha}_{2}+\hat{\beta}_{2} \bar{u}_{1}^{2}\right)-\left(\hat{\alpha}_{1}+\hat{\beta}_{1} \bar{u}_{1}^{2}\right)\left(\hat{\alpha}_{2}+\hat{\beta}_{2} \bar{u}_{2}^{2}\right)\right]}{\left(1+\bar{u}_{1}^{2}+\bar{u}_{2}^{2}\right)^{2}\left(\bar{\alpha}_{1}+\bar{\beta}_{1} \bar{u}_{1}^{2}\right)\left(\bar{\alpha}_{2}+\bar{\beta}_{2} \bar{u}_{2}^{2}\right)} .
\end{aligned}
$$

We now can see that the trace is negative and the determinant is positive if $\bar{u}_{1}$ is small and $\bar{u}_{2}$ is large or if $\bar{u}_{1}$ is large and $\bar{u}_{2}$ is small. Hence, if $\mathcal{N}>E$, for some large $E$, the two steady states are stable.

Having understood the steady-state behavior of systems (2.4)-(2.6) for extreme values of the copy number, we now investigate what changes can happen to the steady states as we vary $\mathcal{N}$ from 0 to $\infty$. First, we recall a few useful concepts and theorems.

Definition 3.3. Let $U \subset \mathbb{R}^{n}, V \subset \mathbb{R}^{m}$ be open, and let $f: U \rightarrow V$ be a smooth function. A point $x \in U$ is called a regular point of $f$ if the derivative of $f$ at $x, D_{x} f$, is a surjective linear map. Otherwise, it is called a critical point. A point $y \in V$ is called a regular value of $f$ if $f^{-1}(y)$ does not contain critical points. Otherwise, it is a critical value of $f$.

A classical result, which is a corollary of the Inverse Function Theorem, states that if $y \in V$ is a regular value of $f$, then $f^{-1}(y)$ is a smooth manifold of dimension $n-m$. In particular, it is a smooth curve when $m=n-1$.

Another classical result that will be useful in our analysis is the following theorem.

Theorem 3.4 (Bendixson-Dulac). Consider a plane autonomous system

$$
\begin{aligned}
& \dot{x}=f(x, y), \\
& \dot{y}=g(x, y) .
\end{aligned}
$$

Copyright (c) by SIAM. Unauthorized reproduction of this article is prohibited. 
Suppose that $f$ and $g$ are continuously differentiable in an open and simply connected region $U \subset \mathbb{R}^{2}$. If there exists a continuously differentiable in $U$ function $\varphi(x, y)$ such that

$$
\frac{\partial(\varphi f)}{\partial x}+\frac{\partial(\varphi g)}{\partial y} \neq 0
$$

almost everywhere in $U$, then the above system does not have periodic solutions in $U$.

Now, we are ready to get back to systems (2.4)-(2.6).

Lemma 3.5. The following hold:

1. The origin is a regular value of the function $F_{p f}$.

2. If $\bar{\alpha}_{1} \bar{\beta}_{1} \neq \bar{\alpha}_{2} \bar{\beta}_{2}$, then the origin is a regular value of the function $F_{b f}$.

3. Let

$$
U_{ \pm}(a)=\frac{1}{\sqrt{2}} \sqrt{a-3 \pm \sqrt{(a-3)^{2}-4 a}}
$$

and

$$
v(x, a)=\frac{x\left(a+x^{2}\right)}{1+x^{2}} .
$$

Also, let $a_{i}=\frac{\bar{\alpha}_{i}}{\bar{\beta}_{i}}, i=1,2$. If the values $\bar{\beta}_{1} v\left(U_{ \pm}\left(a_{1}\right), a_{1}\right)$ do not coincide with any of the values $\bar{\beta}_{2} v\left(U_{ \pm}\left(a_{2}\right), a_{2}\right)$, that is, if

$$
\left\{\bar{\beta}_{1} v\left(U_{-}\left(a_{1}\right), a_{1}\right), \bar{\beta}_{1} v\left(U_{+}\left(a_{1}\right), a_{1}\right)\right\} \cap\left\{\bar{\beta}_{2} v\left(U_{-}\left(a_{2}\right), a_{2}\right), \bar{\beta}_{2} v\left(U_{+}\left(a_{2}\right), a_{2}\right)\right\}=\emptyset,
$$

then the origin is a regular value of the function $F_{t s}$.

Proof. Notice that

$$
\frac{\partial F_{p f}}{\partial \mathcal{N}}=-1 \neq 0
$$

which proves the first statement.

Now consider

$$
\frac{\partial F_{b f}}{\partial \mathcal{N}}=(-1,0)
$$

Thus, $F_{b f}$ can have a critical point only if

$$
\operatorname{grad}\left(g_{b f}\right)=\left(\frac{\bar{\alpha}_{1}-\bar{\beta}_{1} \bar{u}_{1}^{2}}{\left(\bar{\alpha}_{1}+\bar{\beta}_{1} \bar{u}_{1}^{2}\right)^{2}}, \frac{\bar{\alpha}_{2}-\bar{\beta}_{2} \bar{u}_{2}^{2}}{\left(\bar{\alpha}_{2}+\bar{\beta}_{2} \bar{u}_{2}^{2}\right)^{2}}\right)=0 .
$$

This equation holds only if $\bar{u}_{1}=\sqrt{\frac{\bar{\alpha}_{1}}{\bar{\beta}_{1}}}$ and $\bar{u}_{2}=\sqrt{\frac{\bar{\alpha}_{2}}{\bar{\beta}_{2}}}$. But $g\left(\sqrt{\frac{\bar{\alpha}_{1}}{\bar{\beta}_{1}}}, \sqrt{\frac{\bar{\alpha}_{2}}{\beta_{2}}}\right)=0$ only if $\bar{\alpha}_{1} \bar{\beta}_{1}=\bar{\alpha}_{2} \bar{\beta}_{2}$, which proves the second statement.

Similarly,

$$
\frac{\partial F_{t s}}{\partial \mathcal{N}}=(-1,0)
$$

and

$$
\operatorname{grad}\left(g_{t s}\right)=\left(\frac{\bar{u}_{1}^{4}+\left(3-a_{2}\right) \bar{u}_{1}^{2}+a_{2}}{\left(1+\bar{u}_{1}^{2}\right)^{2}},-\frac{\bar{u}_{2}^{4}+\left(3-a_{1}\right) \bar{u}_{2}^{2}+a_{1}}{\left(1+\bar{u}_{2}^{2}\right)^{2}}\right) .
$$

Copyright $\odot$ by SIAM. Unauthorized reproduction of this article is prohibited. 
It follows that the gradient of $g_{t s}$ is zero iff

$$
\begin{aligned}
& \bar{u}_{1}=U_{ \pm}\left(a_{2}\right), \\
& \bar{u}_{2}=U_{ \pm}\left(a_{1}\right) .
\end{aligned}
$$

Notice that

$$
g_{t s}\left(\bar{u}_{1}, \bar{u}_{2}\right)=\bar{\beta}_{2} v\left(\bar{u}_{1}, a_{2}\right)-\bar{\beta}_{1} v\left(\bar{u}_{2}, a_{1}\right) .
$$

Since $\left(\bar{u}_{1}, \bar{u}_{2}\right) \in g_{t s}^{-1}(0)$, and $\operatorname{grad}\left(g_{t s}\right)=0$ implies (3.10) and (3.11), we obtain that the origin can be a critical value of $F_{t s}$ only if at least one of the values $\bar{\beta}_{2} v\left(U_{ \pm}\left(a_{2}\right), a_{2}\right)$ coincides with one of $\bar{\beta}_{1} v\left(U_{ \pm}\left(a_{1}\right), a_{1}\right)$.

The above lemma immediately leads to the following corollary.

Corollary 3.6. For each of the systems (2.4)-(2.6) the set of all steady states obtained by varying $\mathcal{N}$ is a smooth curve. This curve is embedded in $\mathbb{R}^{2}$ for the case of the positive feedback circuit and in $\mathbb{R}^{3}$ for the other two cases.

It is not difficult to describe the structure of the steady state curves in more detail. For example, it follows directly from (3.1) that the curve of steady states for the positive feedback circuit is the graph of the function $f_{p f}$ (see Figure 2(a),(b)).

For the bistable feedback circuit, notice that the equation $g_{b f}=0$ defines a curve, $C_{b f}$, in the $\left(\bar{u}_{1}, \bar{u}_{2}\right)$ plane. The steady-state curve is then the graph of the function $f_{b f}$ restricted to the curve $C_{b f}$ (see Figure 2(c),(d)). Notice that $C_{b f}$, and hence the curve of steady states, has two unbounded connected components, one of which contains the origin. Indeed, these components are given by the graphs of the functions $h_{ \pm}$from the proof of Theorem 3.2 if $\bar{\alpha}_{1} \bar{\beta}_{1}>\bar{\alpha}_{2} \bar{\beta}_{2}$ (the corresponding functions for the other case can be obtained in a similar way). As we shall discuss later, the component located away from the origin may represent biologically unattainable steady states; the other component always corresponds to biologically relevant states. Looking back at Theorem 3.2, we can see that for large values of $\mathcal{N}$ one of the stable steady states belongs to the component containing the origin, while the other two steady states reside on the other component.

The case of the toggle switch is quite similar, as shown in Figure 2(e),(f). Again, $g_{t s}=0$ defines a curve, $C_{t s}$, in the $\left(\bar{u}_{1}, \bar{u}_{2}\right)$ plane, and the curve of steady states is the graph of $\left.f_{t s}\right|_{C_{t s}}$. The curve $C_{t s}$ might also have two components. In such a case, one of the components is a closed curve, and the other is an unbounded curve containing the origin. Both components, however, give biologically relevant steady states, and therefore we do not distinguish between them. It is also clear that the stable steady state from Theorems 3.1 and 3.2 belongs to the unbounded component.

The above results suggest the following picture. At low values of the copy number we have a single stable node that corresponds to the intersection of the plane $\mathcal{N}=$ small const with the curve of steady states. As $\mathcal{N}$ increases, the number of intersection points (and hence steady states) may change (Figure 2(a),(c),(e)), which will lead to a drastic change in the equilibrium dynamics. Such an event corresponds to a saddle-node bifurcation. The only other generic way for our systems to drastically change their equilibrium dynamics is a Hopf bifurcation. Fortunately, it turns out that the latter case cannot happen in systems (2.4)-(2.6).

Theorem 3.7. Systems (2.4)-(2.6) cannot have periodic orbits. 

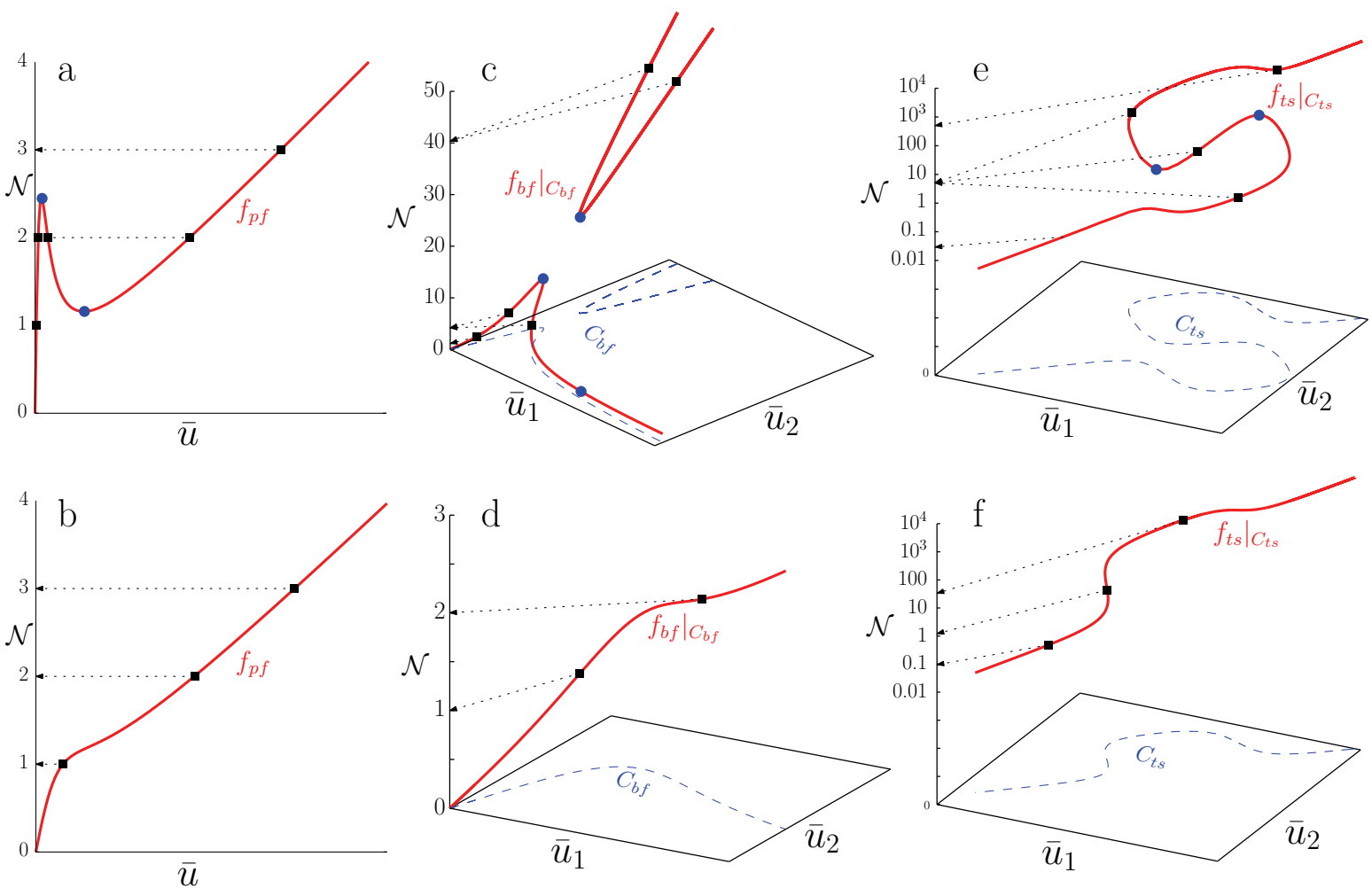

Figure 2. Examples of steady state curves for the positive feedback circuit (a)-(b), bistable feedback circuit (c)-(d), and toggle switch circuit (e)-(f). The top row shows the case when bifurcations due to CNV are present (the corresponding parameter values are $(\bar{\alpha}, \bar{\beta})=(0.025,1.7),\left(\bar{\alpha}_{1}, \bar{\beta}_{1}, \bar{\alpha}_{2}, \bar{\beta}_{2}\right)=(0.05,1.5,0.6667,0.1083)$, and $\left(\bar{\alpha}_{1}, \bar{\beta}_{1}, \bar{\alpha}_{2}, \bar{\beta}_{2}\right)=(5.9125,0.0137,4.333,0.0433)$, respectively $)$; the bottom row shows the case when bifurcations would not occur (the corresponding parameter values are $(\bar{\alpha}, \bar{\beta})=(0.25,1.3),\left(\bar{\alpha}_{1}, \bar{\beta}_{1}, \bar{\alpha}_{2}, \bar{\beta}_{2}\right)=$ $(0.375,0.875,0.6667,0.1083)$, and $\left(\bar{\alpha}_{1}, \bar{\beta}_{1}, \bar{\alpha}_{2}, \bar{\beta}_{2}\right)=(0.9625,0.0137,2.1667,0.0433)$, respectively $)$. Bifurcation points are marked by blue circles. Projections of some steady states (marked by black squares) onto the $\mathcal{N}$-axis are shown by dotted arrows.

Proof. The statement is trivial for system (2.4). In the case of system (2.6) the result follows from the Bendixson-Dulac theorem. Simply notice that the divergence of the righthand side is $-\left(\gamma_{1}+\gamma_{2}\right) \neq 0$.

The case of system (2.5) requires a little more work. First notice that multiplying the right-hand side of (2.5) by $1+u_{1}^{2}+u_{2}^{2}$ yields a topologically conjugate system (see, e.g., [22]):

$$
\dot{u}_{i}=\mathcal{N}\left(\hat{\alpha}_{i}+\hat{\beta}_{i} u_{i}^{2}\right)-\gamma_{i} u_{i}\left(1+u_{1}^{2}+u_{2}^{2}\right), \quad i=1,2 .
$$

Now make the following change of variables: $u_{i}=\sqrt{\gamma_{i}} v_{i}$. Then we obtain

$$
\dot{v}_{i}=h_{i}\left(v_{1}, v_{2}\right)=\mathcal{N}\left(\frac{\hat{\alpha}_{i}}{\sqrt{\gamma_{i}}}+\hat{\beta}_{i} \sqrt{\gamma_{i}} v_{i}^{2}\right)-\gamma_{i} v_{i}\left(1+\gamma_{1} v_{1}^{2}+\gamma_{2} v_{2}^{2}\right), \quad i=1,2 .
$$

Since

$$
\frac{\mathrm{d} h_{1}}{\mathrm{~d} v_{2}}=-\gamma_{1} \gamma_{2} v_{1} v_{2}=\frac{\mathrm{d} h_{2}}{\mathrm{~d} v_{1}}
$$

Copyright (c) by SIAM. Unauthorized reproduction of this article is prohibited. 
the above system is, in fact, a gradient system.

Thus, employing the classical result of [2], we can now state that changes in equilibrium dynamics of systems (2.4)-(2.6) may occur only via saddle-node bifurcations. Such a restriction on the type of possible bifurcations simplifies our goal: now we need only find conditions that guarantee (non)existence of saddle-node bifurcations. The following lemma will be very helpful in this endeavor.

Lemma 3.8. Consider a smooth map $f(x, \mu), f: \mathbb{R}^{n+p} \rightarrow \mathbb{R}^{n}, x \in \mathbb{R}^{n}, \mu \in \mathbb{R}^{p}$. Suppose that 0 is a regular value of $f$, and let $M=f^{-1}(0)$. Then $D_{x} f\left(x_{0}, \mu_{0}\right),\left(x_{0}, \mu_{0}\right) \in M$ is singular iff $\mu_{0}$ is a critical value of the projection map $\pi: M \rightarrow \mathbb{R}^{p}$ given by $\pi(x, \mu)=\mu$.

Proof. First, suppose that $D_{x} f\left(x_{0}, \mu_{0}\right)$ is not singular. Then it follows from the Implicit Function Theorem that there are neighborhoods $U \subset \mathbb{R}^{n+p}$ and $V \subset \mathbb{R}^{p}$ of $\left(x_{0}, \mu_{0}\right)$ and $\mu_{0}$, respectively, such that $M \cap U$ is a graph of a smooth function $g: V \rightarrow \mathbb{R}^{n}$. Consequently, $\mu_{0}$ cannot be a critical value of $\pi$.

Now suppose that $\mu_{0}$ is a regular value of $\pi$. Then $\pi$ is a local diffeomorphism. Therefore, $v=(y, 0) \notin T_{\left(x_{0}, \mu_{0}\right)} M$ if $y \neq 0$. Since $\operatorname{ker}\left(D f\left(x_{0}, \mu_{0}\right)\right)=T_{\left(x_{0}, \mu_{0}\right)} M$, we obtain $D f\left(x_{0}, \mu_{0}\right) v=$ $D_{x} f\left(x_{0}, \mu_{0}\right) y \neq 0$ for all $y \neq 0$. Hence, $D_{x}\left(x_{0}, \mu_{0}\right)$ is nonsingular.

4. Conditions for existence of saddle-node bifurcations. Figure $2(\mathrm{a}),(\mathrm{c}),(\mathrm{e})$ shows that systems (2.4)-(2.6) may undergo saddle-node bifurcations as we vary the copy number. A different choice of parameter values results in a picture with no bifurcations (see Figure $2(\mathrm{~b}),(\mathrm{d}),(\mathrm{f}))$. We shall now derive conditions on the parameters in systems (2.4)-(2.6) that guarantee existence (and absence) of saddle-node bifurcations caused by variation in the copy number.

4.1. Positive feedback motif. It follows directly from Lemma 3.8 that saddle-node bifurcations in system (2.4) which are caused by changes in the copy number correspond to critical values of the map $f_{p f}$. Consequently, we can prove the following theorem.

Theorem 4.1. If $\beta>9 \alpha$, then system (2.4) undergoes two consecutive saddle-node bifurcations as $\mathcal{N}$ varies from 0 to $\infty$. If $\beta<9 \alpha$, the bifurcations will not occur.

Proof. We need to find out when equation $\frac{\partial f_{p f}}{\partial \bar{u}}=0$, that is,

$$
\frac{\bar{\beta} \bar{u}^{4}-(\bar{\beta}-3 \bar{\alpha}) \bar{u}^{2}+\bar{\alpha}}{\left(\bar{\alpha}+\bar{\beta} \bar{u}^{2}\right)^{2}}=0,
$$

has a solution. Since the denominator is always greater than zero, we get the biquadratic equation

$$
\bar{u}^{4}-(1-3 a) \bar{u}^{2}+a=0
$$

where $a=\frac{\bar{\alpha}}{\beta}=\frac{\alpha}{\beta}$. Solving for $\bar{u}^{2}$, we find

$$
\bar{u}^{2}=\frac{1}{2}\left(1-3 a \pm \sqrt{(1-3 a)^{2}-4 a}\right) .
$$

We are interested in simple, positive real roots. Therefore, we need

$$
\begin{aligned}
1-3 a & >0, \\
(1-3 a)^{2}-4 a & >0 .
\end{aligned}
$$

Copyright $\odot$ by SIAM. Unauthorized reproduction of this article is prohibited. 
The solution to these inequalities is given by $a<\frac{1}{9}$ or, equivalently, $\beta>9 \alpha$. Thus, if this condition is satisfied, then there will be two saddle-node bifurcations at

$$
\mathcal{N}_{ \pm}=\frac{\bar{u}_{ \pm}\left(1+\bar{u}_{ \pm}^{2}\right)}{\bar{\alpha}+\bar{\beta} \bar{u}_{ \pm}^{2}}
$$

where

$$
\bar{u}_{ \pm}=\frac{1}{\sqrt{2}} \sqrt{1-3 a \pm \sqrt{(1-3 a)^{2}-4 a}} .
$$

It is clear that if $\beta<9 \alpha$, then (4.1) has no solution, so function $f_{p f}$ has no critical points, and no bifurcations can occur.

Thus, as long as the enhanced transcription rate is sufficiently greater than the basal transcription rate, $\mathrm{CNV}$ of the positive feedback circuit may lead to drastic changes in its expression dynamics. The value of the copy number at which the sharp transition occurs is a tunable feature that will have important consequences for how the entire system responds to CNV. In particular, if this value is too large or too low, it may be of no biological relevance.

4.2. Toggle switch motif. In this case the map $\pi$ from Lemma 3.8 becomes the projection map onto the $\mathcal{N}$-axis, as shown in Figure 2(e),(f). Critical points of this map are critical points of the map $f_{t s}$ restricted to the curve $g_{t s}=0$ (see (3.6), (3.7)). Therefore, we have to look for critical points of the corresponding Lagrangian. Interestingly, the result we obtain is very similar to Theorem 4.1.

Theorem 4.2. If $\alpha_{i}>9 \beta_{i}, i=1,2$, and

$$
\left(\bar{\beta}_{1} v\left(U_{+}\left(a_{1}\right), a_{1}\right), \bar{\beta}_{1} v\left(U_{-}\left(a_{1}\right), a_{1}\right)\right) \cap\left(\bar{\beta}_{2} v\left(U_{+}\left(a_{2}\right), a_{2}\right), \bar{\beta}_{2} v\left(U_{-}\left(a_{2}\right), a_{2}\right)\right) \neq \emptyset,
$$

where

$$
\begin{gathered}
v(x, a)=\frac{x\left(a+x^{2}\right)}{1+x^{2}} \\
U_{ \pm}(a)=\frac{1}{\sqrt{2}} \sqrt{a-3 \pm \sqrt{(a-3)^{2}-4 a}}
\end{gathered}
$$

and $a_{i}=\frac{\bar{\alpha}_{i}}{\beta_{i}}, i=1,2$, then system (2.6) undergoes two consecutive saddle-node bifurcations as we vary $\mathcal{N}$ from 0 to $\infty$. If $\alpha_{i}<9 \beta_{i}, i=1,2$, then the bifurcations will not occur.

Proof. Without loss of generality we can assume that

$$
\bar{\beta}_{2} v\left(U_{-}\left(a_{2}\right), a_{2}\right)<\bar{\beta}_{1} v\left(U_{-}\left(a_{1}\right), a_{1}\right) .
$$

The case of the opposite inequality is proved by swapping the indices and then applying the same argument.

We need to find out when function $f_{t s}$ restricted to the curve $g_{t s}=0$ has critical points. To simplify the algebra, we shall modify the above problem by replacing $f_{t s}$ and $g_{t s}$ with their logarithms, $f_{l t s}$ and $g_{l t s}$, respectively. That is, we look for extrema of

$$
f_{l t s}\left(\bar{u}_{1}, \bar{u}_{2}\right)=\log \bar{u}_{1}+\log \left(1+\bar{u}_{2}^{2}\right)-\log \left(\bar{\alpha}_{1}+\bar{\beta}_{1} \bar{u}_{2}^{2}\right)
$$


restricted to the curve

$$
g_{\text {lts }}\left(\bar{u}_{1}, \bar{u}_{2}\right)=\log \left(\frac{\bar{u}_{1}\left(\bar{\alpha}_{2}+\bar{\beta}_{2} \bar{u}_{1}^{2}\right)}{1+\bar{u}_{1}^{2}}\right)-\log \left(\frac{\bar{u}_{2}\left(\bar{\alpha}_{1}+\bar{\beta}_{1} \bar{u}_{2}^{2}\right)}{1+\bar{u}_{2}^{2}}\right)=0 .
$$

The Lagrangian for this problem is given by

$$
L\left(\bar{u}_{1}, \bar{u}_{2}, \lambda\right)=f_{l t s}\left(\bar{u}_{1}, \bar{u}_{2}\right)+\lambda g_{l t s}\left(\bar{u}_{1}, \bar{u}_{2}\right) .
$$

The partial derivatives of $L\left(\bar{u}_{1}, \bar{u}_{2}, \lambda\right)$ are

$$
\begin{aligned}
\frac{\partial L}{\partial \bar{u}_{1}} & =\frac{(1+\lambda) \bar{u}_{1}^{4}+\left[(1+\lambda)\left(1+a_{2}\right)+2 \lambda\left(1-a_{2}\right)\right] \bar{u}_{1}^{2}+(1+\lambda) a_{2}}{\bar{u}_{1}\left(1+\bar{u}_{1}^{2}\right)\left(a_{2}+\bar{u}_{1}^{2}\right)}, \\
\frac{\partial L}{\partial \bar{u}_{2}} & =\frac{-\lambda \bar{u}_{2}^{4}+\left[2(1+\lambda)\left(a_{1}-1\right)-\lambda\left(1+a_{1}\right)\right] \bar{u}_{2}^{2}-\lambda a_{1}}{\bar{u}_{2}\left(1+\bar{u}_{2}^{2}\right)\left(a_{1}+\bar{u}_{2}^{2}\right)}, \\
\frac{\partial L}{\partial \lambda} & =\log \left(\frac{\bar{\beta}_{2} \bar{u}_{1}\left(a_{2}+\bar{u}_{1}^{2}\right)}{1+\bar{u}_{1}^{2}}\right)-\log \left(\frac{\bar{\beta}_{1} \bar{u}_{2}\left(a_{1}+\bar{u}_{2}^{2}\right)}{1+\bar{u}_{2}^{2}}\right) .
\end{aligned}
$$

Equating these derivatives to zero, we obtain the system

$$
\begin{aligned}
0 & =\bar{u}_{1}^{4}-h\left(\delta, a_{2}\right) \bar{u}_{1}^{2}+a_{2}, \\
0 & =\bar{u}_{1}^{4}-h\left(\delta^{-1}, a_{1}\right) \bar{u}_{1}^{2}+a_{1}, \\
\bar{\beta}_{2} v\left(\bar{u}_{1}, a_{2}\right) & =\bar{\beta}_{1} v\left(\bar{u}_{2}, a_{1}\right),
\end{aligned}
$$

where $\delta=\frac{\lambda}{1+\lambda}$ and

$$
h(x, a)=2 x(a-1)-a-1 .
$$

From (4.3), (4.4) we find

$$
\begin{aligned}
& \bar{u}_{1}=H_{ \pm}\left(\delta, a_{2}\right), \\
& \bar{u}_{2}=H_{ \pm}\left(\delta^{-1}, a_{1}\right),
\end{aligned}
$$

where

$$
H_{ \pm}(x, a)=\frac{1}{\sqrt{2}} \sqrt{h(x, a) \pm \sqrt{h^{2}(x, a)-4 a}} .
$$

We need nondegenerate, real positive solutions for $\bar{u}_{1}$ and $\bar{u}_{2}$. Therefore, the following conditions should be satisfied:

$$
h\left(\delta^{ \pm 1}, a_{i}\right)>0, \quad h^{2}\left(\delta^{ \pm 1}, a_{i}\right)-4 a_{i}>0 .
$$

Solving this system of inequalities, we find that

$$
\frac{\sqrt{a_{2}}+1}{2\left(\sqrt{a_{2}}-1\right)}<\delta<\frac{2\left(\sqrt{a_{1}}-1\right)}{\sqrt{a_{1}}+1} .
$$

Let us denote the left side of the above inequality by $\delta_{l}$ and the right side by $\delta_{r}$. Notice that $\delta_{l}<1$ if $a_{2}>9$ and $\delta_{r}>1$ if $a_{1}>9$. Thus, conditions of the theorem guarantee a 
nonempty range of values for $\delta$. Notice also that if $a_{i}<9, i=1,2$, then inequality (4.6) cannot be satisfied; hence there cannot be any critical points, which proves the second part of the theorem.

System (4.3)-(4.5) will have a solution if we can find $\delta \in\left(\delta_{l}, \delta_{r}\right)$ such that

$$
\bar{\beta}_{2} v\left(H_{ \pm}\left(\delta, a_{2}\right), a_{2}\right)=\bar{\beta}_{1} v\left(H_{ \pm}\left(\delta^{-1}, a_{1}\right), a_{1}\right) .
$$

Notice that $U_{ \pm}\left(a_{i}\right)=H_{ \pm}\left(1, a_{i}\right)$ and that $h(x, a)$ is an increasing function of each of its arguments. Therefore, $H_{+}(x, a)$ is an increasing function of each of its arguments and $H_{-}(x, a)$ is a decreasing function of each of its arguments. Using these observations and the fact that

$$
h\left(\delta_{l}, a_{2}\right)=2 \sqrt{a_{2}}, \quad h\left(\delta_{r}^{-1}, a_{1}\right)=2 \sqrt{a_{1}},
$$

we find that

$$
\begin{gathered}
U_{-}\left(a_{2}\right)<H_{-}\left(\delta_{l}, a_{2}\right)=H_{+}\left(\delta_{l}, a_{2}\right)<U_{+}\left(a_{2}\right), \\
U_{-}\left(a_{1}\right)<H_{-}\left(\delta_{r}^{-1}, a_{1}\right)=H_{+}\left(\delta_{r}^{-1}, a_{1}\right)<U_{+}\left(a_{1}\right),
\end{gathered}
$$

and

$$
\begin{gathered}
H_{-}\left(\delta_{r}, a_{2}\right)<U_{-}\left(a_{2}\right)<U_{+}\left(a_{2}\right)<H_{+}\left(\delta_{r}, a_{2}\right), \\
H_{-}\left(\delta_{l}^{-1}, a_{1}\right)<U_{-}\left(a_{1}\right)<U_{+}\left(a_{1}\right)<H_{+}\left(\delta_{l}^{-1}, a_{1}\right) .
\end{gathered}
$$

A simple calculation shows that $U_{-}(a)$ is a local maximum and $U_{+}(a)$ is a local minimum of $v(x, a)$ (regarded as a function of only its first argument). Consequently, $v(x, a)$ is increasing on $\left(0, U_{-}(a)\right) \cup\left(U_{+}(a), \infty\right)$ and decreasing on $\left(U_{-}(a), U+(a)\right)$. Now recall that

$$
\bar{\beta}_{2} v\left(U_{-}\left(a_{2}\right), a_{2}\right)<\bar{\beta}_{1} v\left(U_{-}\left(a_{1}\right), a_{1}\right) .
$$

Suppose also that

$$
\bar{\beta}_{2} v\left(U_{+}\left(a_{2}\right), a_{2}\right)>\bar{\beta}_{1} v\left(U_{+}\left(a_{1}\right), a_{1}\right)
$$

so that condition (4.2) is satisfied. Then at $\delta=1$ we have

$$
\begin{aligned}
\bar{\beta}_{1} v\left(H_{-}\left(1, a_{1}\right), a_{1}\right)>\bar{\beta}_{2} v\left(H_{-}\left(1, a_{2}\right), a_{2}\right) \\
>\bar{\beta}_{2} v\left(H_{+}\left(1, a_{2}\right), a_{2}\right)>\bar{\beta}_{1} v\left(H_{+}\left(1, a_{1}\right), a_{1}\right) .
\end{aligned}
$$

At $\delta=\delta_{r}$ we have either

$$
\bar{\beta}_{1} v\left(H_{-}\left(\delta_{r}^{-1}, a_{1}\right), a_{1}\right)<\bar{\beta}_{2} v\left(H_{-}\left(\delta_{r}, a_{2}\right), a_{2}\right)
$$

or

$$
\bar{\beta}_{1} v\left(H_{+}\left(\delta_{r}^{-1}, a_{1}\right), a_{1}\right)>\bar{\beta}_{2} v\left(H_{-}\left(\delta_{r}, a_{2}\right), a_{2}\right) .
$$

In either case, there exists $\delta_{1} \in\left(1, \delta_{r}\right)$ such that (4.7) holds. The value of $\bar{u}_{1}$ at this critical point is given by $\bar{u}_{1}=H_{-}\left(\delta_{1}, a_{2}\right)$. The value of $\bar{u}_{2}$ is given by either $\bar{u}_{2}=H_{+}\left(\delta_{1}^{-1}, a_{1}\right)$ or $\bar{u}_{2}=H_{-}\left(\delta_{1}^{-1}, a_{1}\right)$. 
Since $\delta=\delta_{r}$ yields

$$
\bar{\beta}_{1} v\left(H_{-}\left(\delta_{r}^{-1}, a_{1}\right), a_{1}\right)<\bar{\beta}_{2} v\left(H_{+}\left(\delta_{r}, a_{2}\right), a_{2}\right)
$$

or

$$
\bar{\beta}_{1} v\left(H_{+}\left(\delta_{r}^{-1}, a_{1}\right), a_{1}\right)>\bar{\beta}_{2} v\left(H_{+}\left(\delta_{r}, a_{2}\right), a_{2}\right),
$$

there also exists $\delta_{2} \in\left(1, \delta_{r}\right)$ such that (4.7) holds. The value of $\bar{u}_{1}$ at this critical point is given by $\bar{u}_{1}=H_{+}\left(\delta_{2}, a_{2}\right)$. The value of $\bar{u}_{2}$ is given by either $\bar{u}_{2}=H_{+}\left(\delta_{2}^{-1}, a_{1}\right)$ or $\bar{u}_{2}=H_{-}\left(\delta_{2}^{-1}, a_{1}\right)$.

Now suppose that

$$
\bar{\beta}_{2} v\left(U_{+}\left(a_{2}\right), a_{2}\right)<\bar{\beta}_{1} v\left(U_{+}\left(a_{1}\right), a_{1}\right)<\bar{\beta}_{2} v\left(U_{-}\left(a_{2}\right), a_{2}\right),
$$

which corresponds to the other possible case covered by (4.2). Then at $\delta=1$ we have

$$
\begin{aligned}
\bar{\beta}_{1} v\left(H_{-}\left(1, a_{1}\right), a_{1}\right)>\bar{\beta}_{2} v\left(H_{-}\left(1, a_{2}\right), a_{2}\right) \\
>\bar{\beta}_{1} v\left(H_{+}\left(1, a_{1}\right), a_{1}\right)>\bar{\beta}_{2} v\left(H_{+}\left(1, a_{2}\right), a_{2}\right) .
\end{aligned}
$$

At $\delta=\delta_{r}$ we have either

$$
\bar{\beta}_{1} v\left(H_{-}\left(\delta_{r}^{-1}, a_{1}\right), a_{1}\right)<\bar{\beta}_{2} v\left(H_{-}\left(\delta_{r}, a_{2}\right), a_{2}\right)
$$

or

$$
\bar{\beta}_{1} v\left(H_{+}\left(\delta_{r}^{-1}, a_{1}\right), a_{1}\right)>\bar{\beta}_{2} v\left(H_{-}\left(\delta_{r}, a_{2}\right), a_{2}\right) .
$$

Therefore, there exists $\delta_{3} \in\left(1, \delta_{r}\right)$ such that (4.7) holds. The value of $\bar{u}_{1}$ at this critical point is given by $\bar{u}_{1}=H_{-}\left(\delta_{1}, a_{2}\right)$. The value of $\bar{u}_{2}$ is given by either $\bar{u}_{2}=H_{+}\left(\delta_{3}^{-1}, a_{1}\right)$ or $\bar{u}_{2}=H_{-}\left(\delta_{3}^{-1}, a_{1}\right)$.

At $\delta=\delta_{l}$ we have either

$$
\bar{\beta}_{2} v\left(H_{-}\left(\delta_{l}, a_{2}\right), a_{2}\right)<\bar{\beta}_{1} v\left(H_{+}\left(\delta_{l}^{-1}, a_{1}\right), a_{1}\right)
$$

or

$$
\bar{\beta}_{2} v\left(H_{+}\left(\delta_{l}, a_{2}\right), a_{2}\right)>\bar{\beta}_{1} v\left(H_{+}\left(\delta_{l}^{-1}, a_{1}\right), a_{1}\right) .
$$

Consequently, there exists $\delta_{4} \in\left(\delta_{l}, 1\right)$ such that (4.7) holds. The value of $\bar{u}_{1}$ at this critical point is given by either $\bar{u}_{1}=H_{-}\left(\delta_{4}, a_{2}\right)$ or $\bar{u}_{1}=H_{+}\left(\delta_{4}, a_{2}\right)$. The value of $\bar{u}_{2}$ is given by $\bar{u}_{2}=H_{+}\left(\delta_{4}^{-1}, a_{1}\right)$.

Focusing on the necessary part of the conditions for existence of bifurcations in the above theorem, we see that $\mathrm{CNV}$ of the toggle switch circuit can lead to drastic changes in its expression dynamics only if the basal transcription rate is sufficiently greater than the inhibited transcription rate. While the other part of the conditions (which has to hold to guarantee existence of qualitative changes) is difficult to interpret biologically, notice that it holds for a wide range of parameter values and hence is likely to be satisfied in practice. 
4.3. Bistable feedback motif. The map $\pi$ from Lemma 3.8 is once again the projection map onto the $\mathcal{N}$-axis (see Figure 2(c),(d)), and critical points of this map are critical points of the map $f_{b f}$ restricted to the curve defined by $g_{b f}=0$ (see (3.3), (3.4)). However, existence of another unbounded component of the curve of steady states leads to a more complicated behavior.

Theorem 4.3. Let $C_{1}$ and $C_{2}$ denote the two unbounded connected components of the curve of steady states of system (2.5), and let $C_{1}$ be the component containing the origin.

1. If

$$
\beta_{1}>9 \alpha_{1} \quad \text { and } \quad \bar{\alpha}_{1} \bar{\beta}_{1}>\bar{\alpha}_{2} \bar{\beta}_{2}
$$

or

$$
\beta_{2}>9 \alpha_{2} \quad \text { and } \quad \bar{\alpha}_{2} \bar{\beta}_{2}>\bar{\alpha}_{1} \bar{\beta}_{1},
$$

then varying $\mathcal{N}$ from 0 to $\infty$ causes system (2.5) to undergo two consecutive saddlenode bifurcations whose corresponding steady states belong to $C_{1}$. If

$$
\beta_{1}<9 \alpha_{1} \quad \text { and } \quad \bar{\alpha}_{1} \bar{\beta}_{1}>\bar{\alpha}_{2} \bar{\beta}_{2}
$$

or

$$
\beta_{2}<9 \alpha_{2} \quad \text { and } \quad \bar{\alpha}_{2} \bar{\beta}_{2}>\bar{\alpha}_{1} \bar{\beta}_{1},
$$

then there will be no bifurcations on $C_{1}$.

2. Varying $\mathcal{N}$ from 0 to $\infty$ always causes system (2.5) to undergo at least one saddlenode bifurcation whose corresponding steady states belong to $C_{2}$. Moreover, there exists $\varepsilon>0$ such that if

$$
\alpha_{i}<\varepsilon \beta_{i}, \quad i=1,2
$$

then at least three such bifurcations will occur.

Proof. Without loss of generality we can assume that $\bar{\alpha}_{1} \bar{\beta}_{1}>\bar{\alpha}_{2} \bar{\beta}_{2}$. The case when $\bar{\alpha}_{2} \bar{\beta}_{2}>\bar{\alpha}_{1} \bar{\beta}_{1}$ is proved by swapping the indices and then applying the same argument.

First, we need to find out when the Lagrangian

$$
L\left(\bar{u}_{1}, \bar{u}_{2}, \lambda\right)=f_{b f}\left(\bar{u}_{1}, \bar{u}_{2}\right)+\lambda g_{b f}\left(\bar{u}_{1}, \bar{u}_{2}\right)
$$

has critical points. The partial derivatives of $L$ are given by

$$
\begin{aligned}
\frac{\partial L}{\partial \bar{u}_{1}} & =\frac{\bar{\beta}_{1} \bar{u}_{1}^{4}+\left(3 \bar{\alpha}_{1}-(1+\lambda) \bar{\beta}_{1}\right) \bar{u}_{1}^{2}+(1+\lambda) \bar{\alpha}_{1}}{\left(\bar{\alpha}_{1}+\bar{\beta}_{1} \bar{u}_{1}^{2}\right)^{2}}, \\
\frac{\partial L}{\partial \bar{u}_{2}} & =\frac{\bar{\beta}_{2} \bar{u}_{2}^{4}+\left(3 \bar{\alpha}_{2}+\lambda \bar{\beta}_{2}\right) \bar{u}_{2}^{2}-\lambda \bar{\alpha}_{2}}{\left(\bar{\alpha}_{2}+\bar{\beta}_{2} \bar{u}_{2}^{2}\right)^{2}}, \\
\frac{\partial L}{\partial \lambda} & =g\left(\bar{u}_{1}, \bar{u}_{2}\right)=\frac{\bar{u}_{1}}{\bar{\alpha}_{1}+\bar{\beta}_{1} \bar{u}_{1}^{2}}-\frac{\bar{u}_{2}}{\bar{\alpha}_{2}+\bar{\beta}_{2} \bar{u}_{2}^{2}} .
\end{aligned}
$$

Copyright ( $\odot$ by SIAM. Unauthorized reproduction of this article is prohibited. 
Equating them to zero, we get the system of equations

$$
\begin{aligned}
& 0=\bar{u}_{1}^{4}-\left((1+\lambda)-3 a_{1}\right) \bar{u}_{1}^{2}+(1+\lambda) a_{1}, \\
& 0=\bar{u}_{2}^{4}+\left(\lambda+3 a_{2}\right) \bar{u}_{2}^{2}-\lambda a_{2}, \\
& 0=\frac{\bar{u}_{1}}{\bar{\alpha}_{1}+\bar{\beta}_{1} \bar{u}_{1}^{2}}-\frac{\bar{u}_{2}}{\bar{\alpha}_{2}+\bar{\beta}_{2} \bar{u}_{2}^{2}},
\end{aligned}
$$

where $a_{i}=\frac{\bar{\alpha}_{i}}{\beta_{i}}, i=1,2$. Notice that the first two of the above equations are biquadratic. Solving them with respect to $\bar{u}_{1}^{2}$ and $\bar{u}_{2}^{2}$, we find that $\bar{u}_{1}^{2}=a_{1} v_{1, \pm}(\lambda)$, and $\bar{u}_{2}^{2}=a_{2} v_{2, \pm}(\lambda)$, where

$$
\begin{aligned}
& v_{1, \pm}(\lambda)=\frac{1}{2}\left(b_{1}(\lambda)-3 \pm \sqrt{\left(b_{1}(\lambda)-3\right)^{2}-4 b_{1}(\lambda)}\right), \\
& v_{2, \pm}(\lambda)=\frac{1}{2}\left(-\left(b_{2}(\lambda)+3\right) \pm \sqrt{\left(b_{2}(\lambda)+3\right)^{2}+4 b_{2}(\lambda)}\right),
\end{aligned}
$$

and $b_{1}(\lambda)=\frac{1+\lambda}{a_{1}}, b_{2}(\lambda)=\frac{\lambda}{a_{2}}$. We are interested in simple real positive roots, and some of $v_{i, \pm}(\lambda), i=1,2$, can be negative or complex, depending on the values of $\lambda$. We need to distinguish three cases.

Case $1: \lambda>0$. In this case $v_{2,-}(\lambda)$ is always negative and $v_{2,+}(\lambda)$ is always positive. $v_{1, \pm}(\lambda)$ is real and positive when $b_{1}(\lambda)-3>0$ and $\left(b_{1}(\lambda)-3\right)^{2}-4 b_{1}(\lambda)>0$. Solving these inequalities leads to the condition $b_{1}(\lambda)>9$.

Since we are looking for positive $\bar{u}_{1}$ and $\bar{u}_{2},(4.16)$ is equivalent to

$$
\frac{\bar{u}_{1}^{2}}{\bar{\beta}_{1}^{2}\left(a_{1}+\bar{u}_{1}^{2}\right)^{2}}=\frac{\bar{u}_{2}^{2}}{\bar{\beta}_{2}^{2}\left(a_{2}+\bar{u}_{2}^{2}\right)^{2}} .
$$

Substituting expressions for $\bar{u}_{1}^{2}$ and $\bar{u}_{2}^{2}$ and performing several algebraic manipulations, we get

$$
\bar{\alpha}_{2} \bar{\beta}_{2} h\left(v_{1, \pm}(\lambda)\right)=\bar{\alpha}_{1} \bar{\beta}_{1} h\left(v_{2,+}(\lambda)\right),
$$

where $h(x)=\frac{x}{(1+x)^{2}}$. If there is $\lambda>0$ such that the two sides are equal, then system (4.14)(4.16) has a solution determined by such a $\lambda$. The conditions of the theorem state that $a_{1}<9$, which implies that $b_{1}(\lambda)>9$ for all $\lambda>0$. We shall now show that there are $\lambda_{1}>0$ and $\lambda_{2}>0$ such that $\bar{\alpha}_{2} \bar{\beta}_{2} h\left(v_{1,+}\left(\lambda_{1}\right)\right)=\bar{\alpha}_{1} \bar{\beta}_{1} h\left(v_{2,+}\left(\lambda_{1}\right)\right)$, and $\bar{\alpha}_{2} \bar{\beta}_{2} h\left(v_{1,-}\left(\lambda_{2}\right)\right)=\bar{\alpha}_{1} \bar{\beta}_{1} h\left(v_{2,+}\left(\lambda_{2}\right)\right)$.

Notice that function $h(x)$ is increasing for $x \in(0,1)$, attains its maximum at $x=1$, and is decreasing for $x \in(1, \infty)$. Computing derivatives of $v_{1, \pm}$ and $v_{2,+}$, we find that

$$
\begin{gathered}
\frac{\partial v_{1, \pm}}{\partial \lambda}=\frac{1}{2 a_{1}}\left(1 \pm \frac{b_{1}-5}{\sqrt{\left(b_{1}-3\right)^{2}-4 b_{1}}}\right), \\
\frac{\partial v_{2,+}}{\partial \lambda}=\frac{1}{2 a_{2}}\left(-1+\frac{b_{2}+5}{\sqrt{\left(b_{2}+3\right)^{2}+4 b_{2}}}\right) .
\end{gathered}
$$

After a few calculations we find that the fractions in the parentheses are greater than 1 if $\lambda>0$. Therefore, $v_{1,+}$ and $v_{2,+}$ are increasing functions of $\lambda$, and $v_{1,-}$ is a decreasing function 
of $\lambda$. In addition, we find that $v_{1,-}(\lambda) \rightarrow 1, v_{2,+}(\lambda) \rightarrow 1$, and $v_{1,+}(\lambda) \rightarrow \infty$ as $\lambda \rightarrow \infty$. Also, $v_{1, \pm}(0)>1$ and $v_{2,+}(0)=0$. Thus, when we increase $\lambda$ from 0 to $\infty, v_{2,+}(\lambda)$ will increase from 0 to 1 , and therefore the right-hand side of (4.18) will increase from 0 to its maximum value, $\frac{\bar{\alpha}_{1} \bar{\beta}_{1}}{4}$. At the same time, $v_{1,+}$ will increase from some value greater than 1 to infinity. Therefore, the corresponding left-hand side will decrease from a positive value to zero. Since the changes are continuous, there will be a value of $\lambda=\lambda_{1}$ such that the two sides are equal. Similarly, $v_{1,-}$ will decrease from some positive value greater than 1 to 1 . The corresponding left-hand side will therefore increase to its maximum value, $\frac{\bar{\alpha}_{2} \bar{\beta}_{2}}{4}$. Recall, however, that $\bar{\alpha}_{1} \bar{\beta}_{1}>\bar{\alpha}_{2} \bar{\beta}_{2}$. Therefore, the maximum of the right-hand side of (4.18) is strictly greater than the maximum of the left-hand side, which implies that at some $\lambda=\lambda_{2}$ the two sides will become equal.

Thus, if conditions (4.10) are satisfied, system (4.14)-(4.16) has two solutions for $\lambda>0$. The first solution is given by $\lambda=\lambda_{1}, u_{1}=\sqrt{a_{1} v_{1,+}\left(\lambda_{1}\right)}$, and $u_{2}=\sqrt{a_{2} v_{2,+}\left(\lambda_{1}\right)}$. The second is given by $\lambda=\lambda_{2}, u_{1}=\sqrt{a_{1} v_{1,-}\left(\lambda_{2}\right)}$, and $u_{2}=\sqrt{a_{2} v_{2,+}\left(\lambda_{2}\right)}$. We shall show later that the corresponding bifurcations occur on $C_{1}$, which proves the first statement of the theorem.

Case 2: $-1<\lambda<0$. In this case both $v_{2, \pm}$ are positive if $b_{2}(\lambda)<-9 . v_{1, \pm}$ is positive when $b_{1}(\lambda)>9$. To simplify analysis, we assume that $a_{i}<18, i=1,2$, which implies that the above conditions are satisfied for $\lambda \in\left(9 a_{1}-1,-9 a_{2}\right)$. Similarly to the case $\lambda>0$, we can show that $v_{1,+}$ and $v_{2,-}$ are increasing functions of $\lambda$, and $v_{1,-}$ and $v_{2,+}$ are decreasing functions of $\lambda$. Notice that $v_{1, \pm}\left(9 a_{1}-1\right)=3$ and $v_{2, \pm}\left(-9 a_{2}\right)=3$. Also, $b_{1}\left(-9 a_{2}\right)=\frac{1-9 a_{2}}{a_{1}}$ and $-b_{2}\left(9 a_{1}-1\right)=\frac{1-9 a_{1}}{a_{2}}$. These values are very large if $a_{1}$ and $a_{2}$ are very small. Therefore, choosing sufficiently small $a_{1}$ and $a_{2}$, we can make $v_{1,-}\left(-9 a_{2}\right)$ and $v_{2,-}\left(9 a_{1}-1\right)$ arbitrarily close to 1 and $v_{1,+}\left(-9 a_{2}\right)$ and $v_{2,+}\left(9 a_{1}-1\right)$ arbitrarily large.

Recall that $\bar{\alpha}_{1} \bar{\beta}_{1}>\bar{\alpha}_{2} \bar{\beta}_{2}$ and that 1 is the only maximum of $h(x)$ for $x>0$. Also, $\bar{\alpha}_{1} \bar{\beta}_{1}<\frac{8}{3} \bar{\alpha}_{2} \bar{\beta}_{2}$ implies that $\bar{\alpha}_{2} \bar{\beta}_{2} h(1)>\bar{\alpha}_{1} \bar{\beta}_{1} h(3)$. Assume that this inequality holds. Then if $a_{1}$ and $a_{2}$ are sufficiently small, we have $\bar{\alpha}_{2} \bar{\beta}_{2} h\left(v_{1,-}\left(-9 a_{2}\right)\right)>\bar{\alpha}_{1} \bar{\beta}_{1} h\left(v_{2,-}\left(-9 a_{2}\right)\right)$ and $\bar{\alpha}_{2} \bar{\beta}_{2} h\left(v_{1,-}\left(9 a_{1}-1\right)\right)<\bar{\alpha}_{1} \bar{\beta}_{1} h\left(v_{2,-}\left(9 a_{1}-1\right)\right)$. Since dependence on $\lambda$ in the above expressions is continuous, there is $\lambda_{3} \in\left(9 a_{1}-1,-9 a_{2}\right)$ such that the two sides are equal. If $\bar{\alpha}_{1} \bar{\beta}_{1} \geq \frac{8}{3} \bar{\alpha}_{2} \bar{\beta}_{2}$, then $\bar{\alpha}_{2} \bar{\beta}_{2} h\left(v_{1,-}\left(-9 a_{2}\right)\right)<\bar{\alpha}_{1} \bar{\beta}_{1} h\left(v_{2,+}\left(-9 a_{2}\right)\right)$ but $\bar{\alpha}_{2} \bar{\beta}_{2} h\left(v_{1,-}\left(9 a_{1}-1\right)\right)>$ $\bar{\alpha}_{1} \bar{\beta}_{1} h\left(v_{2,+}\left(9 a_{1}-1\right)\right)$, so again there exists $\lambda_{3} \in\left(9 a_{1}-1,-9 a_{2}\right)$ such that the two sides are equal. Regardless of the sign of $\bar{\alpha}_{1} \bar{\beta}_{1}-\frac{8}{3} \bar{\alpha}_{2} \bar{\beta}_{2}, \bar{\alpha}_{2} \bar{\beta}_{2} h\left(v_{1,+}\left(-9 a_{2}\right)\right)<\bar{\alpha}_{1} \bar{\beta}_{1} h\left(v_{2,+}\left(-9 a_{2}\right)\right)$ and $\bar{\alpha}_{2} \bar{\beta}_{2} h\left(v_{1,+}\left(9 a_{1}-1\right)\right)>\bar{\alpha}_{1} \bar{\beta}_{1} h\left(v_{2,+}\left(9 a_{1}-1\right)\right)$ for sufficiently small $a_{1}$ and $a_{2}$. Therefore, there is $\lambda_{4} \in\left(9 a_{1}-1,-9 a_{2}\right)$ such that the latter two sides are equal.

Thus, we have shown that if $a_{i} \ll 1$ or, equivalently, $\alpha_{i} \ll \beta_{i}, i=1,2$, then system (4.14)(4.16) has two solutions for $\lambda \in(-1,0)$. The first is given by $\lambda=\lambda_{3}, u_{1}=\sqrt{a_{1} v_{1,-}\left(\lambda_{3}\right)}$, and $u_{2}=\sqrt{a_{2} v_{2,-}\left(\lambda_{3}\right)}$, or $u_{2}=\sqrt{a_{2} v_{2,+}\left(\lambda_{3}\right)}$. The second is given by $\lambda=\lambda_{4}, u_{1}=\sqrt{a_{1} v_{1,+}\left(\lambda_{4}\right)}$, and $u_{2}=\sqrt{a_{2} v_{2,+}\left(\lambda_{4}\right)}$.

Case 3: $\lambda<-1$. In this case $v_{1,-}$ is always negative and $v_{1,+}$ is always positive. $v_{2, \pm}$ are positive when $b_{2}(\lambda)<-9$. Repeating steps from the previous cases, we can show that as $\lambda$ decreases from $-9 a_{2}$ (or -1 , whichever is less) to $-\infty, v_{1,+}$ increases from a nonnegative value $V_{1}<1$ to $1, v_{2,-}$ decreases from a positive value $V_{2}>1$ to 1 , and $v_{2,+}$ increases from a positive value $V_{3} \geq V_{2}$ to infinity. Recall that $\bar{\alpha}_{1} \bar{\beta}_{1}>\bar{\alpha}_{2} \bar{\beta}_{2}$, and notice that

$$
\bar{\alpha}_{2} \bar{\beta}_{2} h\left(v_{1,+}(-\Lambda)\right)>\bar{\alpha}_{1} \bar{\beta}_{1} h\left(v_{2,+}(-\Lambda)\right),
$$


and

$$
\bar{\alpha}_{2} \bar{\beta}_{2} h\left(v_{1,+}(-\Lambda)\right)<\bar{\alpha}_{1} \bar{\beta}_{1} h\left(v_{2,-}(-\Lambda)\right),
$$

where $\Lambda>0$ is sufficiently large. If $-9 a_{2}>-1$, we start decreasing $\lambda$ from -1 , so $V_{1}=0<$ $V_{2}$. Therefore, $\bar{\alpha}_{2} \bar{\beta}_{2} h\left(v_{1,+}(-1)\right)<\bar{\alpha}_{1} \bar{\beta}_{1} h\left(v_{2,+}(-1)\right)$, and it follows from (4.19) that there is $\lambda_{5}<-1$ such that the above two sides are equal. If $-9 a_{2}<-1$, we start decreasing $\lambda$ from $-9 a_{2}$, so $V_{1}>0$ and $V_{2}=V_{3}=3$. Depending on the sign of $\bar{\alpha}_{2} \bar{\beta}_{2} h\left(V_{1}\right)-\bar{\alpha}_{1} \bar{\beta}_{1} h(3)$, we employ (4.20) or (4.19) to show that there still is $\lambda_{5}<-1$ such that the corresponding sides are equal.

Thus, there always is a solution to system (4.14)-(4.16) for $\lambda<-1$ given by $\lambda=\lambda_{5}$, $u_{1}=\sqrt{a_{1} v_{1,+}\left(\lambda_{5}\right)}$, and $u_{2}=\sqrt{a_{2} v_{2,+}\left(\lambda_{5}\right)}$ or $u_{2}=\sqrt{a_{2} v_{2,-}\left(\lambda_{5}\right)}$.

We shall now determine the branch, $C_{1}$ or $C_{2}$, to which the above critical points belong. Let us denote the curve defined by $g_{b f}\left(\bar{u}_{1}, \bar{u}_{2}\right)=0$ by $c$. The two branches of $c$ are graphs of the functions

$$
\bar{u}_{2}=U_{ \pm}\left(\bar{u}_{1}\right)=\frac{1}{2}\left(w\left(\bar{u}_{1}\right) \pm \sqrt{w^{2}\left(\bar{u}_{1}\right)-4 \frac{\bar{\alpha}_{2}}{\bar{\beta}_{2}}}\right)
$$

where $w\left(\bar{u}_{1}\right)=\frac{\bar{\alpha}_{1}+\bar{\beta}_{1} \bar{u}_{1}^{2}}{\beta_{2} \bar{u}_{1}}$. A simple analysis of the two branches shows that $U_{-}$is increasing on $\left(0, \sqrt{a_{1}}\right)$, has maximum at $\bar{u}_{1}=\sqrt{a_{1}}$, and is decreasing on $\left(\sqrt{a_{1}}, \infty\right)$, while $U_{+}$is decreasing on $\left(0, \sqrt{a_{1}}\right)$, has minimum at $\bar{u}_{1}=\sqrt{a_{1}}$, and is increasing on $\left(\sqrt{a_{1}}, \infty\right)$. Here, as before, $a_{1}=\frac{\bar{\alpha}_{1}}{\beta_{1}}$. We also find that

$$
U_{ \pm}\left(\sqrt{a_{1}}\right)=\frac{1}{\bar{\beta}_{2}}\left(\sqrt{\bar{\alpha}_{1} \bar{\beta}_{1}} \pm \sqrt{\bar{\alpha}_{1} \bar{\beta}_{1}-\bar{\alpha}_{2} \bar{\beta}_{2}}\right) .
$$

Therefore, $U_{-}\left(\bar{u}_{1}\right)<U_{+}\left(\bar{u}_{1}\right)$ for all $\bar{u}_{1}>0$.

Let us denote critical points corresponding to $\lambda_{i}$ by $p_{i}, 1 \leq i \leq 5$. Consider $p_{i}, i=3,4,5$. Notice that at each of these points $\bar{u}_{2}$ is greater than $\sqrt{a_{2}}$. We also have $U_{-}\left(\sqrt{a_{1}}\right)<\sqrt{a_{2}}$ and hence $U_{-}\left(\bar{u}_{1}\right)<\sqrt{a_{2}}$ for all $\bar{u}_{1}>0$. Consequently, the three critical points should lie on the upper branch of the curve $c$. Therefore, the corresponding bifurcations occur on $C_{2}$. To determine the branch of $p_{1}$ and $p_{2}$, recall that we found these points by starting at a point below the lower branch (in fact, on the axis $\bar{u}_{2}=0$ ) and then increasing $\bar{u}_{2}$ until we hit the curve $c$. It is clear that we could hit only the lower branch. Therefore, the first two critical points belong to the lower branch of the curve $c$, and the corresponding bifurcations occur on $C_{1}$.

Interestingly, we once again see that a possibility of qualitative changes in expression dynamics of a gene circuit due to its copy number variation requires a sufficiently large difference between the basal and, in this case, enhanced transcription rates. We also notice again that conditions which need to be added to guarantee existence of the drastic effect hold for a wide range of parameter values.

5. Discussion. We have performed a detailed bifurcation analysis of standard models of three common regulatory gene circuits and demonstrated not only the fact that drastic changes in expression dynamics may be caused by CNV, but also that this phenomenon is robust with respect to the other parameters present in the systems. In particular, we have derived closed form expressions for conditions that guarantee the presence of saddle-node bifurcations in the three dynamical systems. Our analysis provides a detailed description of the behavior of the 
systems as we vary the copy number. In fact, we have shown that all three circuits have a single stable steady state for small values of $\mathcal{N}$. As this parameter increases, all three systems enter a bistable regime when a new stable node and a saddle are created through a saddlenode bifurcation. Increasing the copy number a little further causes the systems to undergo another saddle-node bifurcation which eliminates the old steady state (and the saddle), again causing a sharp transition in equilibrium dynamics. While we have also shown that more saddle-node bifurcations may happen in the bistable feedback circuit as $\mathcal{N}$ increases even further, we should notice that those bifurcations belong to a different connected component of the curve of steady states. This component lies away from the origin and, depending on other parameter values, may correspond only to very large values of the copy number. For example, if $\bar{\beta}_{2}$, which is the (rescaled) transcription rate of a promoter occupied by the second protein, is significantly smaller than the $\bar{\alpha}_{1} \bar{\beta}_{1}$, then the values of $\mathcal{N}$ on this branch will be unrealistically large and biologically irrelevant. But it turns out that such a relation among the parameters of the bistable feedback circuit is quite common; in fact, often $\beta_{2} \approx 0$ (see, e.g., [25]).

For the sake of analytic tractability, the three models of gene circuit dynamics that we have analyzed were derived under the simplifying assumption that dimer degradation rates were negligible. In reality, cell growth leads to dilution of all gene products, including dimers. Nevertheless, the general approach to studying the CNV effect in gene circuits developed in this paper remains applicable even when dimer degradation is taken into account. We have analyzed all three gene circuits in the case when dimer degradation is included (see Appendix B). The corresponding analysis indicates that the qualitative nature of changes in equilibrium dynamics due to CNV remains the same. See Figure 3 for examples of bifurcations in each circuit as a function of $\mathcal{N}$ when dimer degradation is included.

Our analysis was also based on the assumption that CNV was balanced, that is, that variation affected all components of small circuits rather than individual genes. Such an assumption is valid in the case of synthetic and natural systems. In particular, synthetically engineered circuits, simultaneous viral infections, duplications of genome segments, and changes in plasmid number can all lead to balanced variation in the number of gene circuits. However, there are many situations, such as gene duplication, in which CNV may affect single genes, leading to imbalances in components of gene circuits. The authors are actively working on a similar analysis of imbalanced CNV and its effects on gene expression.

As we pointed out in the introduction, we have referred to the bifurcation parameter $\mathcal{N}$ as the "copy number" for convenience; however, it is formally defined as the copy number concentration. Hence, a change in the cell volume can also lead to the foregoing bifurcations. This property can be exploited synthetically as a means to detect cell volume changes. It also suggests that many gene circuits may have evolved to stay sufficiently far from the bifurcation regions. Otherwise, variations in cell volume during the cell cycle would lead to (potentially deteriorating) qualitative changes in expression dynamics. The role of hysteresis in this process may also be important and should be studied.

We should also point out that our analysis was performed for the quasi-steady-state approximations of the original higher dimensional models of circuits' expression dynamics. Most of the theorems that we proved are expected to hold also in the higher dimensional case. In particular, varying the copy number will still produce a smooth curve of steady states for

Copyright (c) by SIAM. Unauthorized reproduction of this article is prohibited. 
each of the systems (see Appendix A). We also have the same behavior for extreme values of the copy number (see Theorems 3.1 and 3.2), although the proof of stability is far more tedious. The crucial pieces that we are missing in the case of higher dimensions are Theorem 3.7 and the result in [2]. Consequently, we cannot eliminate the possibility of other bifurcations as we vary the copy number. For example, a steady state might lose its stability through a Hopf bifurcation and the system might enter an oscillating regime. Moreover, chaotic behavior also cannot be ruled out.

While understanding global dynamics in the higher dimensional case seems hopeless, we should notice that all the theorems from section 4 still hold. In addition, we were able to rule out the possibility of Hopf bifurcations (although not periodic orbits) in systems (2.1) and (2.3). Thus, local behavior of these higher dimensional systems is analogous to that of their quasi-steady-state approximations. Unfortunately, we were not able to obtain such a result for system (2.2), although we believe that it holds. Of course, such local results still do not eliminate the possibility of complex dynamics elsewhere in the phase space. Nevertheless, computer simulations lead us to believe that dynamics of the three higher dimensional systems are qualitatively the same as those of the quasi-steady-state approximations. Hence, we think that results in this paper provide a fairly complete description of the circuits' expression dynamics and dependence of these dynamics on CNV.

The three gene circuits that we have analyzed in this paper are common building blocks of many large regulatory networks. The fact that they exhibit sensitivity to CNV and that such an effect of the copy number is robust indicates that this phenomenon may be an important part of gene regulation. We would like to emphasize again that robustness is crucial in this case. While we were able to obtain our results using classical analytical techniques, we believe that a general tool for solving such problems is long overdue and plan to work in this direction in the future.

Appendix A. Steady-state curves in higher dimensions. Employing the derivation of the quasi-steady-state approximations from [19] it is possible to describe the structure of the steady-state curves for higher dimensional systems (2.1)-(2.3). In fact, these curves are the steady-state curves described in section 3 lifted to the higher dimensional space using the following maps:

$$
\begin{aligned}
y & =c_{p} x^{2} \\
d_{x} & =d \frac{c_{p} c_{d} x^{2}}{1+c_{p} c_{d} x^{2}}, \\
d_{0} & =\frac{d}{1+c_{p} c_{d} x^{2}}, \\
m & =\frac{d}{\gamma_{m}} \frac{\alpha+\beta c_{p} c_{d} x^{2}}{1+c_{p} c_{d} x^{2}}
\end{aligned}
$$

Copyright $\odot$ by SIAM. Unauthorized reproduction of this article is prohibited. 
for the positive feedback circuit,

$$
\begin{aligned}
y_{i} & =c_{p} x_{i}^{2}, \\
d_{0} & =\frac{d}{1+c_{p} c_{d}\left(x_{1}^{2}+x_{2}^{2}\right)}, \\
d_{i} & =d \frac{c_{p} c_{d} x_{i}^{2}}{1+c_{p} c_{d}\left(x_{1}^{2}+x_{2}^{2}\right)}, \\
m_{i} & =\frac{d}{\gamma_{m}} \frac{\alpha_{i}+\beta_{i} c_{p} c_{d} x_{i}^{2}}{1+c_{p} c_{d}\left(x_{1}^{2}+x_{2}^{2}\right)}
\end{aligned}
$$

for the bistable feedback circuit, and

$$
\begin{aligned}
y_{i} & =c_{p} x_{i}^{2} \\
d_{0, i} & =\frac{d}{1+c_{p} c_{d} x_{i+1}^{2}}, \\
d_{i} & =d \frac{c_{p} c_{d} x_{i+1}^{2}}{1+c_{p} c_{d} x_{i+1}^{2}}, \\
m_{i} & =\frac{d}{\gamma_{m}} \frac{\alpha_{i}+\beta_{i} c_{p} c_{d} x_{i+1}^{2}}{1+c_{p} c_{d} x_{i+1}^{2}}
\end{aligned}
$$

for the toggle switch circuit.

Appendix B. Taking into account dimer degradation. When deriving systems (2.1)(2.3) we assumed that the change in dimer concentration due to degradation and dilution is negligible. We now try to investigate how the models and their dynamics change when we drop this assumption.

It is easy to see that incorporating dimer degradation requires only a slight modification to systems (2.1)-(2.3). More precisely, we simply have to add the corresponding degradation term to the equations describing dimer dynamics. Thus, equations describing the positive feedback circuit become

$$
\begin{aligned}
\dot{x} & =2 \kappa_{-} y-2 \kappa_{+} x^{2}+\sigma m-\gamma_{x} x \\
\dot{y} & =-\kappa_{-} y+\kappa_{+} x^{2}+k_{-} d_{y}-k_{+} d_{0} y-\delta y \\
\dot{d_{0}} & =k_{-} d_{y}-k_{+} d_{0} y \\
\dot{d_{y}} & =k_{+} d_{0} y-k_{-} d_{y} \\
\dot{m} & =\alpha d_{0}+\beta d_{y}-\gamma_{m} m,
\end{aligned}
$$

where $\delta$ is the dimer degradation rate and the other parameters are the same as in system (2.1). Equations describing the bistable feedback circuit are

$$
\begin{aligned}
\dot{x}_{i} & =2 \kappa_{-} y_{i}-2 \kappa_{+} x_{i}^{2}+\sigma m_{i}-\gamma_{i} x_{i}, \\
\dot{y}_{i} & =-\kappa_{-} y_{i}+\kappa_{+} x_{i}^{2}+k_{-} d_{i}-k_{+} d_{0} y_{i}-\delta_{i} y_{i}, \\
\dot{d}_{0} & =k_{-}\left(d_{1}+d_{2}\right)-k_{+} d_{0}\left(y_{1}+y_{2}\right), \\
\dot{d}_{i} & =k_{+} d_{0} y_{i}-k_{-} d_{i}, \\
\dot{m}_{i} & =\alpha_{i} d_{0}+\beta_{i} d_{i}-\gamma_{m} m_{i},
\end{aligned}
$$


where $\delta_{i}, i=1,2$, are the dimer degradation rates and the other parameters are the same as in system (2.2). And equations describing the toggle switch circuit are

$$
\begin{aligned}
\dot{x_{i}} & =2 \kappa_{-} y_{i}-2 \kappa_{+} x_{i}^{2}+\sigma m_{i}-\gamma_{i} x_{i}, \\
\dot{y_{i}} & =-\kappa_{-} y_{i}+\kappa_{+} x_{i}^{2}+k_{-} d_{i+1}-k_{+} d_{0, i+1} y_{i}-\delta_{i} y_{i}, \\
\dot{d}_{0, i} & =k_{-} d_{i}-k_{+} d_{0, i} y_{i+1}, \\
\dot{d}_{i} & =k_{+} d_{0, i} y_{i+1}-k_{-} d_{i}, \\
\dot{m}_{i} & =\alpha_{i} d_{0, i}+\beta_{i} d_{i}-\gamma_{m} m_{i},
\end{aligned}
$$

where $\delta_{i}, i=1,2$, are again the dimer degradation rates and the other parameters are the same as in system (2.3).

Once again, using quasi-steady-state approximations, we simplify the above systems to

$$
\begin{gathered}
\dot{u}=\mathcal{N} \frac{\hat{\alpha}+\hat{\beta} u^{2}}{1+u^{2}}-\gamma_{p} u-\hat{\delta} u^{2}, \\
\dot{u}_{i}=\mathcal{N} \frac{\hat{\alpha}_{i}+\hat{\beta}_{i} u_{i}^{2}}{1+u_{1}^{2}+u_{2}^{2}}-\gamma_{i} u_{i}-\hat{\delta}_{i} u_{i}^{2}, \quad i=1,2,
\end{gathered}
$$

and

$$
\dot{u}_{i}=\mathcal{N} \frac{\hat{\alpha}_{i}+\hat{\beta}_{i} u_{i+1}^{2}}{1+u_{i+1}^{2}}-\gamma_{i} u_{i}-\hat{\delta}_{i} u_{i}^{2}, \quad i \in 1+\mathbb{Z}_{2}
$$

respectively. Clearly, the only difference between these and (2.4)-(2.6) is in the additional quadratic degradation term. The rescaling of the variables and parameters is also very similar. Let $c_{p}=\frac{\kappa_{+}}{\kappa_{-}+\delta}, c_{i}=\frac{\kappa_{+}}{\kappa_{-}+\delta_{i}}$, and $c_{d}=\frac{k_{+}}{k_{-}}$. Then

$$
u=\sqrt{c_{p} c_{d}} x, \quad \hat{\alpha}=\frac{C \alpha \sigma \sqrt{c_{p} c_{d}}}{\gamma_{m}}, \quad \hat{\beta}=\frac{C \beta \sigma \sqrt{c_{p} c_{d}}}{\gamma_{m}}, \quad \hat{\delta}=2 \delta \sqrt{\frac{c_{p}}{c_{d}}},
$$

and

$$
u_{i}=\sqrt{c_{i} c_{d}} x_{i}, \quad \hat{\alpha}_{i}=\frac{C \alpha_{i} \sigma \sqrt{c_{i} c_{d}}}{\gamma_{m}}, \quad \hat{\beta}_{i}=\frac{C \beta_{i} \sigma \sqrt{c_{i} c_{d}}}{\gamma_{m}}, \quad \hat{\delta}_{i}=2 \delta_{i} \sqrt{\frac{c_{i}}{c_{d}}} .
$$

Trying to follow the same ideas as before, we (re)define functions $F_{p f}: \mathbb{R}^{2} \rightarrow \mathbb{R}, F_{b f}:$ $\mathbb{R}^{3} \rightarrow \mathbb{R}^{2}$, and $F_{t s}: \mathbb{R}^{3} \rightarrow \mathbb{R}^{2}$ as follows:

$$
F_{p f}(\bar{u}, \mathcal{N})=f_{p f}(\bar{u})-\mathcal{N}, \quad f_{p f}=\frac{h(\bar{u})\left(1+\bar{u}^{2}\right)}{\bar{\alpha}+\bar{\beta} \bar{u}^{2}},
$$

where $\bar{\alpha}=\frac{\hat{\alpha}}{\gamma_{p}}, \bar{\beta}=\frac{\hat{\beta}}{\gamma_{p}}, \bar{\delta}=\frac{\hat{\delta}}{\gamma_{p}}, h(x)=x+\bar{\delta} x^{2}$;

$$
\begin{gathered}
F_{b f}\left(\bar{u}_{1}, \bar{u}_{2}, \mathcal{N}\right)=\left(f_{b f}\left(\bar{u}_{1}, \bar{u}_{2}\right)-\mathcal{N}, g_{b f}\left(\bar{u}_{1}, \bar{u}_{2}\right)\right), \\
f_{b f}\left(\bar{u}_{1}, \bar{u}_{2}\right)=\frac{h_{1}\left(\bar{u}_{1}\right)\left(1+\bar{u}_{1}^{2}\right)}{\bar{\alpha}_{1}+\bar{\beta}_{1} \bar{u}_{1}^{2}}+\frac{h_{2}\left(\bar{u}_{2}\right) \bar{u}_{2}^{2}}{\bar{\alpha}_{2}+\bar{\beta}_{2} \bar{u}_{2}^{2}}, \\
g_{b f}\left(\bar{u}_{1}, \bar{u}_{2}\right)=\frac{h_{1}\left(\bar{u}_{1}\right)}{\bar{\alpha}_{1}+\bar{\beta}_{1} \bar{u}_{1}^{2}}-\frac{h_{2}\left(\bar{u}_{2}\right)}{\bar{\alpha}_{2}+\bar{\beta}_{2} \bar{u}_{2}^{2}},
\end{gathered}
$$

Copyright $\odot$ by SIAM. Unauthorized reproduction of this article is prohibited. 

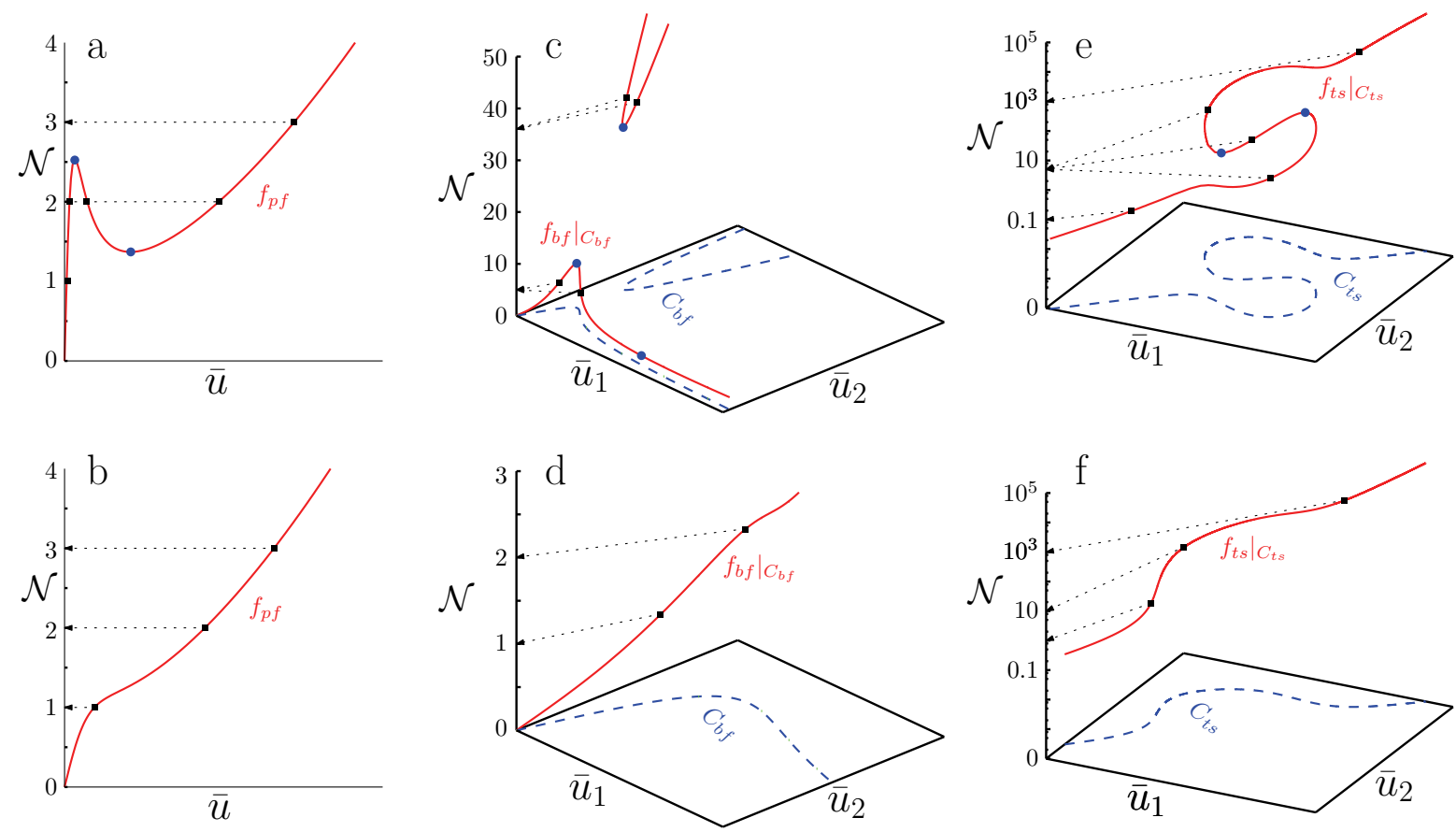

Figure 3. Examples of steady state curves for the positive feedback circuit (a)-(b), bistable feedback circuit (c)-(d), and toggle switch circuit (e)-(f) when dimer degradation/dilution is taken into account. The top row shows the case when bifurcations due to CNV are present (the corresponding parameter values are $\quad(\bar{\alpha}, \bar{\beta}, \bar{\delta})=(0.0249,1.695,0.1995), \quad\left(\bar{\alpha}_{1}, \bar{\beta}_{1}, \bar{\delta}_{1}, \bar{\alpha}_{2}, \bar{\beta}_{2}, \bar{\delta}_{2}\right)=(0.0498,1.494,0.1992,0.6663,0.1082,0.0333)$, and $\left(\bar{\alpha}_{1}, \bar{\beta}_{1}, \bar{\delta}_{1}, \bar{\alpha}_{2}, \bar{\beta}_{2}, \bar{\delta}_{2}\right)=(4.7933,0.0136,0.1992,4.32,0.0432,0.1994), \quad$ respectively $) ; \quad$ bottom row shows the case when bifurcations would not occur (the corresponding parameter values are $(\bar{\alpha}, \bar{\beta}, \bar{\delta})=(0.249,1.496,0.1995), \quad\left(\bar{\alpha}_{1}, \bar{\beta}_{1}, \bar{\delta}_{1}, \bar{\alpha}_{2}, \bar{\beta}_{2}, \bar{\delta}_{2}\right)=(0.3735,0.8715,0.1992,0.6663,0.1082,0.0333), \quad$ and $\left(\bar{\alpha}_{1}, \bar{\beta}_{1}, \bar{\delta}_{1}, \bar{\alpha}_{2}, \bar{\beta}_{2}, \bar{\delta}_{2}\right)=(0.9586,0.0136,0.1992,2.1602,0.0432,0.1994)$, respectively $) . \quad$ Bifurcation points are marked by blue circles. Projections of some steady states (marked by black squares) onto the $\mathcal{N}$-axis are shown by dotted arrows.

where $\bar{\alpha}_{i}=\frac{\hat{\alpha}_{i}}{\gamma_{i}}, \bar{\beta}_{i}=\frac{\hat{\beta}_{i}}{\gamma_{i}}, \bar{\delta}_{i}=\frac{\hat{\delta}_{i}}{\gamma_{i}}, h_{i}(x)=x+\bar{\delta}_{i} x^{2}, i=1,2 ;$ and

$$
\begin{gathered}
F_{t s}\left(\bar{u}_{1}, \bar{u}_{2}, \mathcal{N}\right)=\left(f_{t s}\left(\bar{u}_{1}, \bar{u}_{2}\right)-\mathcal{N}, g_{t s}\left(\bar{u}_{1}, \bar{u}_{2}\right)\right), \\
f_{t s}\left(\bar{u}_{1}, \bar{u}_{2}\right)=\frac{h_{1}\left(\bar{u}_{1}\right)\left(1+\bar{u}_{2}^{2}\right)}{\bar{\alpha}_{1}+\bar{\beta}_{1} \bar{u}_{2}^{2}}, \\
g_{t s}\left(\bar{u}_{1}, \bar{u}_{2}\right)=\frac{h_{1}\left(\bar{u}_{1}\right)\left(\bar{\alpha}_{2}+\bar{\beta}_{2} \bar{u}_{1}^{2}\right)}{1+\bar{u}_{1}^{2}}-\frac{h_{2}\left(\bar{u}_{2}\right)\left(\bar{\alpha}_{1}+\bar{\beta}_{1} \bar{u}_{2}^{2}\right)}{1+\bar{u}_{2}^{2}} .
\end{gathered}
$$

As before, steady states of systems (B.4), (B.5), and (B.6) are solutions to the equations $F_{p f}=0, F_{b f}=0$, and $F_{t s}=0$, respectively.

We shall not try to repeat a complete analysis as presented in sections 3 and 4 here. As one might expect, the general steps remain the same. Instead, we shall only outline the difference (and similarities) in the results.

Statements of Theorems 3.1, 3.2, and 3.7 still hold, and their proofs need only slight

Copyright (C) by SIAM. Unauthorized reproduction of this article is prohibited. 
changes. ${ }^{1}$ The statement of Lemma 3.5, however, becomes more cumbersome. While it is still true that the set of all steady states is, generically, a smooth curve, it is no longer possible to obtain all degeneracy conditions in a closed form. These conditions would have to be described implicitly, and only approximate bounds can be found explicitly. Theorems 4.1, 4.2, and 4.3 present similar difficulties: necessary conditions for existence of bifurcations can be described only implicitly, and (due to approximate bounds) only more crude sufficient conditions can be found.

Despite the above difficulties, we can conclude (and illustrate in Figure 3) that CNV leads to qualitatively the same changes in equilibrium dynamics of the models with and without dimer degradation. Biological conclusions also remain the same: the possibility of drastic changes due to CNV requires sufficiently large differences in the basal and enhanced/inhibited transcription rates, and the presence of the drastic effect is robust with respect to other parameters.

Acknowledgments. We thank Denis Blackmore and Richard Joh for many helpful conversations and two anonymous referees for providing feedback on the manuscript.

\section{REFERENCES}

[1] U. Alon, An Introduction to Systems Biology: Design Principles of Biological Circuits, 1st ed., Chapman \& Hall/CRC, Boca Raton, FL, 2006.

[2] A. Andronov, E. Leontovich, I. Gordon, And A. Maier, Theory of Bifurcations of Dynamical Systems on a Plane, Israel Program of Scientific Translations, Jerusalem, 1971.

[3] W. Ansorge, Next-generation DNA sequencing techniques, New Biotechnology, 25 (2009), pp. $195-203$.

[4] Z. Arai, W. Kalies, H. Kokubu, K. Mischaikow, H. Oka, and P. Pilarczyk, A database schema for the analysis of global dynamics of multiparameter systems, SIAM J. Appl. Dyn. Syst., 8 (2009), pp. 757-789.

[5] V. I. Arnold, Geometrical Methods in the Theory of Ordinary Differential Equations, 2nd ed., Springer, New York, 1996.

[6] V. I. Arnold, Catastrophe Theory, 3rd ed., Springer, New York, 2004.

[7] M. R. Bennett, D. Volfson, L. Tsimring, and J. Hasty, Transient dynamics of genetic regulatory networks, Biophys. J., 92 (2007), pp. 3501-3512.

[8] J. L. Cherry And F. R. Adler, How to make a biological switch, J. Theoret. Biol., 203 (2000), pp. $117-133$.

[9] H. DE Jong, Modeling and simulation of genetic regulatory systems: A literature review, J. Comput. Biol., 9 (2002), pp. 67-103.

[10] T. Ellis, X. Wang, And J. J. Collins, Diversity-based, model-guided construction of synthetic gene networks with predicted functions, Nature Biotechnology, 27 (2009), pp. 465-471.

[11] T. S. Gardner, C. R. Cantor, And J. J. Collins, Construction of a genetic toggle switch in Escherichia coli, Nature, 403 (2000), pp. 339-342.

[12] J. Hasty, J. Pradines, M. Dolnik, and J. J. Collins, Noise-based switches and amplifiers for gene expression, Proc. Natl. Acad. Sci. USA, 97 (2000), pp. 2075-2080.

[13] M. W. Hirsch, S. Smale, And R. Devaney, Differential Equations, Dynamical Systems, and an Introduction to Chaos, 2nd ed., Academic Press, New York, 2003.

[14] F. Isaacs, J. Hasty, C. Cantor, And J. J. Collins, Prediction and measurement of an autoregulatory genetic module, Proc. Natl. Acad. Sci. USA, 100 (2003), pp. 7714-7719.

\footnotetext{
${ }^{1}$ There is one exception. To prove the absence of periodic orbits in system (B.5), we no longer show that the system is conjugate to a gradient system. Rather, we show that it has only real eigenvalues, which, by the way, works for system (2.5) as well.
}

Copyright (c) by SIAM. Unauthorized reproduction of this article is prohibited. 
[15] H. Kim, T. Shay, E. O'Shea, And A. Regev, Transcriptional regulatory circuits: Predicting numbers from alphabets, Science, 325 (2009), pp. 429-432.

[16] Y. A. Kuznetsov, Elements of Applied Bifurcation Theory, 3rd ed., Springer, New York, 2004.

[17] M. Lynch And J. S. Conery, The evolutionary fate and consequences of duplicate genes, Science, 290 (2000), pp. 1151-1155.

[18] N. Metropolis and S. Ulam, The Monte Carlo method, J. Amer. Statist. Assoc., 44 (1949), pp. 335-341.

[19] Y. Mileyko, R. I. Joh, And J. S. Weitz, Small-scale copy number variation and large-scale changes in gene expression, Proc. Natl. Acad. Sci. USA, 105 (2008), pp. 16659-16664.

[20] J. R. Pollack, T. Sørlie, C. M. Perou, C. A. Rees, S. S. Jeffrey, P. E. Lonning, R. Tibshirani, D. Botstein, A. L. BørResen-Dale, And P. Brown, Microarray analysis reveals a major direct role of DNA copy number alteration in the transcriptional program of human breast tumors, Proc. Natl. Acad. Sci. USA, 99 (2002), pp. 12963-12968.

[21] R. Y. Rubinstein And D. P. Kroese, Simulation and the Monte Carlo Method, Wiley Series in Probability and Statistics, Wiley, New York, 2007.

[22] L. P. Shilnikov, A. L. Shilnikov, D. V. Turaev, and L. O. Chua, Methods of Qualitative Theory in Nonlinear Dynamics: Part 1, World Scientific, Singapore, 1998.

[23] L. P. Shilnikov, A. L. Shilnikov, D. V. Turaev, and L. O. Chua, Methods of Qualitative Theory in Nonlinear Dynamics: Part 2, World Scientific, Singapore, 2001.

[24] S. H. Strogatz, Nonlinear Dynamics and Chaos: With Applications to Physics, Biology, Chemistry and Engineering, Perseus Books Group, New York, 2001.

[25] J. S. Weitz, Y. Mileyko, R. I. Joh, And E. O. Voit, Collective decision making in bacterial viruses, Biophys. J., 95 (2008), pp. 2673-2680.

Copyright $($ ) by SIAM. Unauthorized reproduction of this article is prohibited. 